\title{
On dynamic spectral risk measures, a limit theorem and optimal portfolio allocation
}

\author{
D. Madan ${ }^{1} \cdot$ M. Pistorius ${ }^{2} \cdot$ M. Stadje ${ }^{3}$
}

Received: 11 June 2015 / Accepted: 20 March 2017 / Published online: 16 August 2017

(C) The Author(s) 2017. This article is published with open access at Springerlink.com

\begin{abstract}
In this paper, we propose the notion of continuous-time dynamic spectral risk measure (DSR). Adopting a Poisson random measure setting, we define this class of dynamic coherent risk measures in terms of certain backward stochastic differential equations. By establishing a functional limit theorem, we show that DSRs may be considered to be (strongly) time-consistent continuous-time extensions of iterated spectral risk measures, which are obtained by iterating a given spectral risk measure (such as expected shortfall) along a given time-grid. Specifically, we demonstrate that any DSR arises in the limit of a sequence of such iterated spectral risk measures driven by lattice random walks, under suitable scaling and vanishing temporal and spatial mesh sizes. To illustrate its use in financial optimisation problems, we analyse a dynamic portfolio optimisation problem under a DSR.
\end{abstract}

Keywords Spectral risk measure $\cdot$ Dynamic risk measure $\cdot g$-expectation $\cdot$ Choquet expectation · Distortion · (Strong) Time-consistency · Limit theorem · Dynamic portfolio optimisation

Mathematics Subject Classification (2010) $60 \mathrm{H} 10 \cdot 91 \mathrm{~B} 30$

\section{JEL Classification G32}

\section{Pistorius}

m.pistorius@imperial.ac.uk

D. Madan

dbm@rhsmith.umd.edu

M. Stadje

mitja.stadje@uni-ulm.de

1 Robert H. Smith School of Business, University of Maryland, College Park, MD 20742, USA

2 Department of Mathematics, Imperial College London, London SW7 2AZ, UK

3 Faculty of Mathematics and Economics, Universität Ulm, Ulm, Germany 


\section{Introduction}

Financial analysis and decision making rely on quantification and modelling of future risk exposures. A systematic approach for the latter was put forward in [2], laying the foundations of an axiomatic framework for coherent measurement of risk. A subsequent breakthrough was the development and application of the notion of backward stochastic differential equations (BSDEs) in the context of risk analysis, which gave rise to the (strongly) time-consistent extension of coherent risk measures to continuous-time dynamic settings [39, 42]. Building on these advances, we consider in this article a new class of such continuous-time dynamic coherent risk measures, which we propose to call dynamic spectral risk measures (DSRs).

Quantile-based coherent risk measures, such as expected shortfall, belong to the most widely used risk measures in risk analysis, and are also known as spectral risk measures, Choquet expectations (based on probability distortions) and weighted VaR; see $[1,11,34,48]$. In order to carry out for instance an analysis of portfolios involving dynamic rebalancing, one is led to consider the (strongly) time-consistent extension of such coherent risk measures to given time-grids, which are defined by iterative application of the spectral risk measure along these particular grids. Due to its continuous-time domain of definition, a DSR is, in contrast, independent of a grid structure. While the latter holds for any continuous-time risk measure, we show that DSRs emerge as the limits of such iterated spectral risk measures when the time-step vanishes and under appropriate scaling of the parameters, by establishing a functional limit theorem.

To explore its use in financial decision problems, we consider subsequently a dynamic portfolio optimisation problem under DSR, which we analyse in terms of its associated Hamilton-Jacobi-Bellman (HJB) equation. In the case of a long-only investor (who is allowed neither to borrow nor to short-sell stocks), we identify explicitly dynamic optimal allocation strategies.

DSR, like any dynamic risk measure obtained from a BSDE, is (strongly) timeconsistent in the sense that if the value of a random variable $X$ is not larger than $Y$ under DSR at time $t$ almost surely, then the same relation holds at earlier times $s<t$. For dynamic risk measures, the property of strong time-consistency is well known to be equivalent to recursiveness, a tower-type property which is referred to as filtration-consistency in [15]. Such concepts have been investigated extensively in the literature; among others, we mention [3, 10, 14, 16, 25, 30, 31, 40]. For studies on weaker forms of time-consistency, we refer to [41, 47, 49].

The notion of strong time-consistency in economics goes back at least as far as [46] and has been standard in the economics literature ever since; see for instance [ 9 , 20, 23, 24, 27, 32, 33].

Due to their recursive structure, financial optimisation problems, such as utility optimisation under the entropic risk measure and related robust portfolio optimisation problems, satisfy the dynamic programming principle and admit time-consistent dynamically optimal strategies (see for instance [5, 36] and references therein). In Sect. 6, we demonstrate that this also holds for the optimal portfolio allocation problem phrased in terms of the minimisation under a DSR, and phrase and solve this problem via the associated HJB equation. 
For a given DSR, the functional limit theorem that we obtain (see Theorem 5.2) shows how to construct an approximating sequence of iterated spectral risk measures driven by lattice random walks, suggesting an effective method to evaluate functionals under a given DSR and solutions to associated PIDEs, by recursively applying (distorted) Choquet expectations. The functional limit theorem involves a certain non-standard scaling of the parameters of the iterated spectral risk measures, which is given in Definition 5.1. The advantage of this approximation method is that it avoids the (typically nontrivial) task of computing Malliavin derivatives. A numerical study is beyond the scope of the current paper and left for future research.

While one may prove the functional limit theorem directly through duality arguments, we present in the interest of brevity a proof that draws on the convergence results obtained in [37] for weak approximation of BSDEs. In the literature, various related convergence results are available, of which we next mention a number (refer to [37] for additional references). The construction of continuous-time dynamic risk measures arising as limits of discrete-time ones was studied in [45] in a Brownian setting. In a more general setting including in addition finitely many Poisson processes, [35] presents a limit theorem for recursive coherent quantile-based risk measures, which is proved via an associated nonlinear partial differential equation. In [19], a Donsker-type theorem is established under a $G$-expectation.

Contents The remainder of the paper is organised as follows. In Sect. 2, we collect preliminary results concerning dynamic coherent risk measures and related BSDEs, adopting a pure jump setting driven by a Poisson random measure. In Sect. 3, we are concerned with the Choquet-type integrals which appear in the definitions of dynamic and iterated spectral risk measures. With these results in hand, we phrase the definition of a DSR in Sect. 4 and identify its dual representation. In Sect. 5, we present the functional limit theorem for iterated spectral risk measures. Finally, in Sect. 6, we turn to the study of a dynamic portfolio allocation problems under a DSR.

\section{Preliminaries}

In this section, we collect elements of the theory of time-consistent dynamic coherent risk measures and associated BSDEs, in both continuous-time and discrete-time settings. To avoid repetition, we state some results and definitions in terms of the index set $\mathcal{I}$, which is taken to be either $\mathcal{I}=[0, T]$ or

$$
\mathcal{I}=\pi_{\Delta}:=\left\{t_{i}=i \Delta, i=0, \ldots, N\right\}, \quad \text { with } \Delta=T / N,
$$

for some $N \in \mathbb{N}$ and $T>0$.

\subsection{Time-consistent dynamic coherent risk measures}

On some filtered probability space $(\Omega, \mathcal{F}, \mathbf{F}, \mathbb{P})$ with $\mathbf{F}=\left(\mathcal{F}_{t}\right)_{t \in I}$, we consider risks described by random variables $X \in \mathcal{L}^{p}=\mathcal{L}^{p}\left(\mathcal{F}_{T}\right), p>0$, the set of $\mathcal{F}_{T}$-measurable $X$ with $\mathbb{E}\left[|X|^{p}\right]=\int_{\Omega}|X|^{p} \mathrm{~d} P<\infty$. We denote by $\mathcal{L}_{t}^{p}=\mathcal{L}^{p}\left(\mathcal{F}_{t}\right)$ and $\mathcal{L}^{p}(\mathcal{G})$ the elements $X$ in $\mathcal{L}^{p}(\mathcal{F})$ that are measurable with respect to the sigma-algebras $\mathcal{F}_{t}$ and 
$\mathcal{G} \subset \mathcal{F}$, respectively, and by $\mathcal{L}^{\infty}, \mathcal{L}_{t}^{\infty}, \mathcal{L}^{\infty}(\mathcal{G})$ the collections of bounded elements in $\mathcal{L}^{p}, \mathcal{L}_{t}^{p}$ and $\mathcal{L}^{p}(\mathcal{G})$. Let $\mathcal{S}^{2}(\mathcal{I})$ denote the space of $\mathbf{F}$-adapted semimartingales $Y=\left(Y_{t}\right)_{t \in \mathcal{I}}$ that are square-integrable in the sense that $\|Y\|_{\mathcal{S}^{2}(\mathcal{I})}^{2}<\infty$, where

$$
\|Y\|_{\mathcal{S}^{2}(\mathcal{I})}^{2}:=\mathbb{E}\left[\sup _{t \in \mathcal{I}}\left|Y_{t}\right|^{2}\right]
$$

For a given measure $\mu$ on a measurable space $(\mathbb{U}, \mathcal{U})$, we denote by $\mathcal{L}^{p}(\mu), p>0$, the set of Borel functions $v: \mathbb{U} \rightarrow \mathbb{R}$ with $|v|_{p, \mu}<\infty$, where

$$
|v|_{p, \mu}:=\left(\int_{\mathbb{U}}|v(x)|^{p} \mu(\mathrm{d} x)\right)^{1 / p},
$$

and by $\mathcal{L}_{+}^{p}(\mu)$ the set of nonnegative elements in $\mathcal{L}^{p}(\mu)$.

Dynamic coherent risk measures and (strong) time-consistency are defined in an $\mathcal{L}^{2}$-setting as follows.

Definition 2.1 A dynamic coherent risk measure $\rho=\left(\rho_{t}\right)_{t \in I}$ is defined to be a map $\rho: \mathcal{L}^{2} \rightarrow \mathcal{S}^{2}(\mathcal{I})$ that satisfies the following properties:

(i) (cash invariance) for $m \in \mathcal{L}_{t}^{2}, \rho_{t}(X+m)=\rho_{t}(X)-m$;

(ii) (monotonicity) for $X, Y \in \mathcal{L}^{2}$ with $X \geq Y, \rho_{t}(X) \leq \rho_{t}(Y)$;

(iii) (positive homogeneity) for $X \in \mathcal{L}^{2}$ and $\lambda \in \mathcal{L}_{t}^{\infty}, \rho_{t}(|\lambda| X)=|\lambda| \rho_{t}(X)$;

(iv) (subadditivity) for $X, Y \in \mathcal{L}^{2}, \rho_{t}(X+Y) \leq \rho_{t}(X)+\rho_{t}(Y)$.

Definition 2.2 A dynamic coherent risk measure $\rho$ is called (strongly) timeconsistent if either of the following holds:

(v) (strong time-consistency) for $X, Y \in \mathcal{L}^{2}$ and $s, t$ with $s \leq t, \rho_{t}(X) \leq \rho_{t}(Y)$ implies $\rho_{S}(X) \leq \rho_{S}(Y)$;

(vi) (recursiveness) for $X \in \mathcal{L}^{2}$ and $s, t$ with $s \leq t, \rho_{s}\left(\rho_{t}(X)\right)=\rho_{s}(X)$.

More generally, a map $\rho: \mathcal{L}^{2} \rightarrow \mathcal{S}^{2}(\mathcal{I})$ is called a time-consistent dynamic risk measure if $\rho$ satisfies conditions (i) and (v). For a proof of the equivalence of (v) and (vi), we refer to Föllmer and Schied [26, Lemma 11.11]; for a discussion of (the unconditional version of) the properties (i)-(iv), see [2, 3]. One way to construct a timeconsistent dynamic risk measure is as solution to an associated backward stochastic differential equation (BSDE) or backward stochastic difference equation (BS $\Delta \mathrm{E}$ ). To ensure that such dynamic risk measures satisfy (iii) and (iv), the corresponding driver functions must be positively homogeneous, subadditive and should not depend on the value of the risk measure (see [42, Proposition 11] and [15, Lemma 2.1]). Furthermore, a necessary condition to ensure that a comparison principle (and hence the monotonicity in (ii)) holds is that the driver function in addition satisfies a gradient condition (see [17, Theorem 3.2.2]). For background on the notion of strong timeconsistency and its relation to $g$-expectations, we refer to [6, 7, 39, 42]. Specifically, in our setting, such driver functions are defined as follows. 
Definition 2.3 For a given Borel measure $\mu$ on $\mathbb{R}^{k} \backslash\{0\}, k \in \mathbb{N}$, we call a function $g: \mathcal{I} \times \mathcal{L}^{2}(\mu) \rightarrow \mathbb{R}$ a driver function if for any $z \in \mathcal{L}^{2}(\mu)$, the mapping $t \mapsto g(t, z)$ is continuous (in case $\mathcal{I}=[0, T]$ ) and the following holds:

(i) (Lipschitz-continuity) for some $K \in \mathbb{R}_{+} \backslash\{0\}$ and any $t \in \mathcal{I}$ and $z_{1}, z_{2} \in \mathcal{L}^{2}(\mu)$,

$$
\left|g\left(t, z_{1}\right)-g\left(t, z_{2}\right)\right| \leq K\left|z_{1}-z_{2}\right|_{2, \mu}
$$

A driver function $g$ is called coherent if the following hold:

(ii) (positive homogeneity) for any $r \in \mathbb{R}_{+}, t \in \mathcal{I}$ and $z \in \mathcal{L}^{2}(\mu)$, we have

$$
g(t, r z)=r g(t, z)
$$

(iii) (subadditivity) for any $t \in \mathcal{I}$ and $z_{1}, z_{2} \in \mathcal{L}^{2}(\mu)$, we have

$$
g\left(t, z_{1}+z_{2}\right) \leq g\left(t, z_{1}\right)+g\left(t, z_{2}\right) .
$$

(iv) (gradient condition) for any $t \in \mathcal{I}$ and $z_{1}, z_{2} \in \mathcal{L}^{2}(\mu)$, we have

$$
g\left(t, z_{1}\right)-g\left(t, z_{2}\right) \leq \int_{\mathbb{R}^{k} \backslash\{0\}} \delta^{z_{1}, z_{2}}(t, x)\left(z_{1}(x)-z_{2}(x)\right) \mu(\mathrm{d} x),
$$

where the mapping $\delta^{z_{1}, z_{2}}:[0, T] \times \mathbb{R}^{k} \backslash\{0\} \rightarrow(-1, \infty)$ is such that the mapping $t \mapsto \int_{\mathbb{R}^{k} \backslash\{0\}}\left|\delta^{z_{1}, z_{2}}(t, x)\right|^{2} \mu(\mathrm{d} x)$ is bounded, uniformly in $\left(z_{1}, z_{2}\right)$.

If a driver function is convex and positively homogeneous, condition (iv) is satisfied if the subgradients of $g$ are bounded, uniformly in $(t, z) \in \mathcal{I} \times \mathcal{L}^{2}(\mu)$.

We describe next the dynamic (coherent) risk measures defined via BSDEs (if $\mathcal{I}=[0, T]$ ) and BS $\Delta$ Es (if $\mathcal{I}$ is a finite partition of $[0, T]$ ).

\subsection{Discrete-time lattice setting}

We turn first to the discrete-time lattice setting, fixing a uniform partition $\pi=\pi_{\Delta}$ of $[0, T]$ with $\Delta=T / N$ for some $N \in \mathbb{N}$. Let $L^{(\pi)}=\left(L_{t}^{(\pi)}\right)_{t \in \pi}$ denote a squareintegrable zero-mean random walk starting at zero and taking values in $(\sqrt{\Delta} \mathbb{Z})^{k}$, and let $\mathbf{F}^{(\pi)}=\left(\mathcal{F}_{t}^{(\pi)}\right)_{t \in \pi}$ denote the filtration generated by $L^{(\pi)}$. Furthermore, we let $g^{(\pi)}$ be a coherent driver function as in Definition 2.3 with $\mathcal{I}=\pi$ and $\mu(\mathrm{d} x)$ equal to the scaled law $v^{(\pi)}(\mathrm{d} x)$ of $\Delta L_{t}^{(\pi)}=L_{t+\Delta}^{(\pi)}-L_{t}^{(\pi)}, t \in \pi \backslash\{T\}$, given by

$$
v^{(\pi)}(\mathrm{d} x):=\frac{1}{\Delta} \mathbb{P}\left[\Delta L_{t}^{(\pi)} \in \mathrm{d} x\right], \quad x \in(\sqrt{\Delta} \mathbb{Z})^{k} .
$$

In view of [37, Proposition 3.2], the BS $\Delta \mathrm{E}$ for $\left(Y^{(\pi)}, Z^{(\pi)}\right)$ corresponding to the final value $-X^{(\pi)} \in \mathcal{L}^{2}\left(\mathcal{F}_{T}^{(\pi)}\right)$ and the driver function $g^{(\pi)}$ takes the form, analogous to the one in the continuous-time case given in (2.6) below, 


$$
\begin{aligned}
Y_{t}^{(\pi)}= & -X^{(\pi)}+\sum_{s=t}^{T-\Delta} g^{(\pi)}\left(s, Z_{s}^{(\pi)}\right) \Delta \\
& -\sum_{s=t}^{T-\Delta}\left(Z_{s}^{(\pi)}\left(\Delta L_{s}^{(\pi)}\right) I_{\left\{\Delta L_{s}^{(\pi)} \neq 0\right\}}-\mathbb{E}\left[Z_{s}^{(\pi)}\left(\Delta L_{s}^{(\pi)}\right) I_{\left\{\Delta L_{s}^{(\pi)} \neq 0\right\}} \mid \mathcal{F}_{s}^{(\pi)}\right]\right)
\end{aligned}
$$

for $t \in \pi \backslash\{T\}$ and with $Y_{T}^{(\pi)}=-X^{(\pi)}$, where $I_{A}$ denotes the indicator of a set $A$. In difference notation, the BS $\Delta \mathrm{E}(2.2)$ is for $t \in \pi \backslash\{T\}$ given by

$$
\begin{aligned}
\Delta Y_{t}^{(\pi)}= & -g^{(\pi)}\left(t, Z_{t}^{(\pi)}\right) \Delta \\
& +Z_{t}^{(\pi)}\left(\Delta L_{t}^{(\pi)}\right) I_{\left\{\Delta L_{t}^{(\pi)} \neq 0\right\}}-\mathbb{E}\left[Z_{t}^{(\pi)}\left(\Delta L_{t}^{(\pi)}\right) I_{\left\{\Delta L_{t}^{(\pi)} \neq 0\right\}} \mid \mathcal{F}_{t}^{(\pi)}\right]
\end{aligned}
$$

with $Y_{T}^{(\pi)}=-X^{(\pi)}$. A pair $\left(Y^{(\pi)}, Z^{(\pi)}\right)$ is a solution of the BS $\Delta \mathrm{E}$ if for any $t \in \pi$, it satisfies (2.2) with

$$
Y_{t}^{(\pi)} \in \mathcal{L}^{2}\left(\mathcal{F}_{t}^{(\pi)}\right), \quad Z_{t}^{(\pi)} \in \mathbb{L}_{t}^{2}:=\mathcal{L}^{2}\left(v^{(\pi)}(\mathrm{d} x) \times \mathrm{d} \mathbb{P}, \mathcal{B}\left((\sqrt{\Delta} \mathbb{Z})^{k}\right) \otimes \mathcal{F}_{t}^{(\pi)}\right) .
$$

If the Lipschitz constant $K=K^{(\pi)}$ of the driver function $g^{(\pi)}$ is strictly smaller than the reciprocal $1 / \Delta$ of the mesh size, we can use [37, Propositions 3.1 and 3.2] and the fact that in the notation of [37] $F^{(\pi)}$ is independent of $W^{(\pi)}$, to conclude that there exists a unique solution $\left(Y^{(\pi)}, Z^{(\pi)}\right)$ to the $\mathrm{BS} \Delta \mathrm{E}$ which satisfies for $t \in \pi$ the relations

$$
\begin{aligned}
Y_{t}^{(\pi)} & =g^{(\pi)}\left(t, Z_{t}^{(\pi)}\right) \Delta+\mathbb{E}\left[Y_{t+\Delta}^{(\pi)} \mid \mathcal{F}_{t}^{(\pi)}\right], \\
Z_{t}^{(\pi)}(x) & =\mathbb{E}\left[Y_{t+\Delta}^{(\pi)} \mid \mathcal{F}_{t}^{(\pi)} \vee\left\{\Delta L_{t}^{(\pi)}=x\right\}\right]-\mathbb{E}\left[Y_{t+\Delta}^{(\pi)} \mid \mathcal{F}_{t}^{(\pi)} \vee\left\{\Delta L_{t}^{(\pi)}=0\right\}\right]
\end{aligned}
$$

for $x \in(\sqrt{\Delta} \mathbb{Z})^{k}$, where $\mathcal{F}_{t}^{(\pi)} \vee\left\{\Delta L_{t}^{(\pi)}=x\right\}:=\mathcal{F}_{t}^{(\pi)} \vee \sigma\left(\left\{\Delta L_{t}^{(\pi)}=x\right\}\right)$ denotes the smallest sigma-algebra containing $\mathcal{F}_{t}^{(\pi)}$ as well as the sigma-algebra $\sigma\left(\left\{\Delta L_{t}^{(\pi)}=x\right\}\right)$ generated by the set $\left\{\Delta L_{t}^{(\pi)}=x\right\}$. In analogy with the continuous-time case (reviewed below), the dynamic risk measure associated to the solution to the BS $\triangle \mathrm{E}$ is defined as follows.

Definition 2.4 For a driver function $g^{(\pi)}$ as in Definition 2.3 with $\mathcal{I}=\pi$ and with $\mu(\mathrm{d} x)=v^{(\pi)}$ and the solution $\left(Y^{(\pi)}, Z^{(\pi)}\right)$ of the corresponding $\operatorname{BS} \Delta \mathrm{E}$ (2.2), we denote by $\rho^{g^{(\pi)}},(\pi)=\left(\rho_{t}^{g^{(\pi)}},(\pi)\right)_{t \in \pi}$ the time-consistent dynamic risk measure given by $\rho_{t}^{g^{(\pi)},(\pi)}: \mathcal{L}^{2}\left(\mathcal{F}_{T}^{(\pi)}\right) \rightarrow \mathcal{L}^{2}\left(\mathcal{F}_{t}^{(\pi)}\right)$ with

$$
\rho_{t}^{g^{(\pi)},(\pi)}(X)=Y_{t}^{(\pi)} .
$$

\subsection{Continuous-time setting}

In the continuous-time case $\mathcal{I}=[0, T]$, we consider risky positions described by random variables $X$ that are measurable with respect to $\mathcal{F}_{T}$, where $\mathbf{F}=\left(\mathcal{F}_{t}\right)_{t \in[0, T]}$ 
denotes the right-continuous and completed filtration generated by a Poisson random measure $N$ on $[0, T] \times \mathbb{R}^{k} \backslash\{0\}$ for some $k \in \mathbb{N}$. We suppose throughout that the associated Lévy measure $v$ satisfies the following condition:

Assumption 2.5 The Lévy measure $v$ associated to the Poisson random measure $N$ has no atoms, and for some $\varepsilon_{0}>0$, we have $\nu_{2+\varepsilon_{0}} \in \mathbb{R}_{+} \backslash\{0\}$, where for $p \geq 0$,

$$
v_{p}:=\int_{\mathbb{R}^{k} \backslash\{0\}}|x|^{p} v(\mathrm{~d} x) .
$$

We denote by $\tilde{N}(\mathrm{~d} t \times \mathrm{d} x)=N(\mathrm{~d} t \times \mathrm{d} x)-v(\mathrm{~d} x) \mathrm{d} t$ the compensated Poisson random measure and by $L=\left(L_{t}\right)_{t \in[0, T]}$ the (column-vector) Lévy process given by

$$
L_{t}=\int_{[0, t] \times \mathbb{R}^{k} \backslash\{0\}} x \tilde{N}(\mathrm{~d} s \times \mathrm{d} x) .
$$

Under Assumption 2.5, we have $\mathbb{E}\left[\left|L_{t}\right|^{2+\varepsilon_{0}}\right]<\infty$ for any $t \in[0, T]$ (see [44, Theorem 25.3]).

Let $\tilde{\mathcal{H}}^{2}$ denote the set of $\tilde{\mathcal{P}}$-measurable square-integrable processes where, with $\mathcal{P}$ denoting the predictable sigma-algebra, $\tilde{\mathcal{P}}=\mathcal{P} \otimes \mathcal{B}\left(\mathbb{R}^{k} \backslash\{0\}\right)$, and let $\mathcal{U}$ denote the Borel sigma-algebra induced by the $L^{2}(\nu(\mathrm{d} x))$-norm. In particular, $U \in \tilde{\mathcal{H}}^{2}$ is such that $\|U\|_{\tilde{\mathcal{H}}^{2}}<\infty$, where

$$
\|U\|_{\tilde{\mathcal{H}}^{2}}:=\mathbb{E}\left[\int_{0}^{T}\left|U_{t}\right|_{2, \nu}^{2} \mathrm{~d} t\right]
$$

Moreover, let $\mathcal{M}_{2}$ denote the set of probability measures $\mathbb{Q}=\mathbb{Q}^{\xi}$ on $\left(\Omega, \mathcal{F}_{T}\right)$ that are absolutely continuous with respect to $\mathbb{P}$ with square-integrable Radon-Nikodým derivative $\xi \in \mathcal{L}_{+}^{2}\left(\mathcal{F}_{T}\right)$, and write $\mathcal{S}^{2}:=\mathcal{S}^{2}[0, T]$.

Let us next consider a coherent driver function $g$ as in Definition 2.3 with $\mu=v$ and $\mathcal{I}=[0, T]$ and fix a final condition $X \in \mathcal{L}^{2}$. The associated BSDE for the pair $(Y, Z) \in \mathcal{S}^{2} \times \tilde{\mathcal{H}}^{2}$ is given by

$$
Y_{t}=-X+\int_{t}^{T} g\left(s, Z_{s}\right) \mathrm{d} s-\int_{(t, T] \times \mathbb{R}^{k} \backslash\{0\}} Z_{S}(x) \tilde{N}(\mathrm{~d} s \times \mathrm{d} x)
$$

for $t \in[0, T]$. This BSDE, as we recall from [4], admits a unique solution. By combining $[38,43,42]$, we have that the $\operatorname{BSDE}(2.6)$ gives rise to a dynamic coherent risk measure as follows.

Definition 2.6 For a given coherent driver function $g$, the corresponding time-consistent dynamic coherent risk measure $\rho^{g}=\left(\rho_{t}^{g}\right)_{t \in[0, T]}: \mathcal{L}^{2} \rightarrow \mathcal{S}^{2}$ is given by

$$
\rho_{t}^{g}(X)=Y_{t},
$$

where $(Y, Z) \in \mathcal{S}^{2} \times \tilde{\mathcal{H}}^{2}$ solves (2.6). 
Remark 2.7 (i) Let $L^{\mathrm{d}}=\left(L_{t}^{\mathrm{d}}\right)_{t \in[0, T]}$ be given by $L_{t}^{\mathrm{d}}=\mathrm{d} t+L_{t}$ for some $\mathrm{d} \in \mathbb{R}^{k}$. For random variables $X \in \mathcal{L}^{2}$ of the form $X=f\left(L_{T}^{\mathrm{d}}\right)$ for some function $f: \mathbb{R}^{k} \rightarrow \mathbb{R}$, the dynamic coherent risk measure $\rho^{g}(X)$ is related to the semilinear PIDE (denoting $\left.\dot{v}=\frac{\partial v}{\partial t}\right)$

$$
\begin{aligned}
\dot{v}(t, x)+\mathcal{G} v(t, x)+g\left(t, \mathcal{D} v_{t, x}\right) & =0, \quad(t, x) \in[0, T) \times \mathbb{R}^{k}, \\
v(T, x) & =-f(x), \quad x \in \mathbb{R}^{k},
\end{aligned}
$$

where $\mathcal{D} v_{t, x}: \mathbb{R}^{k} \rightarrow \mathbb{R}$ and $\mathcal{G} v(t, x)$ are given by $\mathcal{D} v_{t, x}(y)=v(t, x+y)-v(t, x)$ and

$$
\mathcal{G} v(t, x)=\mathrm{d}^{\top} \nabla v(t, x)+\int_{\mathbb{R}^{k} \backslash\{0\}}\left(\mathcal{D} v_{t, x}(y)-\nabla v(t, x)^{\top} y\right) v(\mathrm{~d} y),
$$

where $\nabla v=\left(\frac{\partial v}{\partial x_{1}}, \ldots, \frac{\partial v}{\partial x_{k}}\right)^{\top}$. Specifically, if $v \in C^{1,1}\left([0, T] \times \mathbb{R}^{k}\right)$ solves (2.7) and (2.8) such that $\nabla v(t, x)$ is bounded (uniformly in $(t, x) \in[0, T] \times \mathbb{R}^{k}$ ), then we have the stochastic representation

$$
\begin{aligned}
\rho_{t}^{g}(X) & =\mathbb{E}\left[-f\left(L_{T}^{\mathrm{d}}\right)+\int_{t}^{T} g\left(s, Z_{s}\right) \mathrm{d} s \mid \mathcal{F}_{t}\right]=v\left(t, L_{t}^{\mathrm{d}}\right), \\
Z_{t}(x) & =v\left(t, L_{t-}^{\mathrm{d}}+x\right)-v\left(t, L_{t-}^{\mathrm{d}}\right), \quad x \in \mathbb{R}^{k},
\end{aligned}
$$

with $L_{0-}^{\mathrm{d}}=L_{0}^{\mathrm{d}}$. This nonlinear Feynman-Kac result is shown by an application of Itô's lemma.

(ii) The risk measure $\rho^{g}$ admits a dual representation

$$
\rho_{t}^{g}(X)=\underset{\mathbb{Q} \in \mathcal{S}^{g}}{\operatorname{ess} \sup } \mathbb{E}^{\mathbb{Q}}\left[-X \mid \mathcal{F}_{t}\right]
$$

for a certain representing subset $\mathcal{S}^{g}$ of the set $\mathcal{M}_{1}$ of probability measures that are absolutely continuous with respect to $\mathbb{P}$. The set $\mathcal{S}^{g}$ is convex and closed (see [26, Theorem 11.22]).

We describe next a representation result for a dynamic risk measure $\rho^{g}$ in terms of the representing processes $\left(H^{\xi}\right)$ of the stochastic logarithms of the Radon-Nikodým derivatives $\xi \in L_{+}^{2}\left(\mathcal{F}_{T}\right)$ of the measures $\mathbb{Q}^{\xi} \in \mathcal{M}_{2}$, which are given by

$$
\xi=\mathcal{E}\left(M^{\xi}\right)_{T}, \quad M^{\xi}=\int_{(0, \cdot] \times \mathbb{R}^{k} \backslash\{0\}} H_{s}^{\xi}(x) \tilde{N}(\mathrm{~d} s \times \mathrm{d} x),
$$

where $\mathcal{E}(\cdot)$ denotes the Doléans-Dade stochastic exponential. A $\mathcal{B}\left(\mathbb{R}^{d}\right) \otimes \mathcal{U}$-measurable set family $C=\left(C_{t}\right)_{t \in[0, T]}$ is called convex or closed if for any $t \in[0, T]$, the set $C_{t}$ is convex or closed.

Theorem 2.8 Let $g$ be a coherent driver function. Then for some $\mathcal{P} \otimes \mathcal{U}$-measurable set family $C^{g}$ that is closed, convex and contains 0 , we have for any $t \in[0, T]$ that 
$\rho_{t}^{g}(X)$ satisfies (2.9) with

$$
\mathcal{S}^{g}=\left\{\mathbb{Q}^{\xi} \in \mathcal{M}_{1}: H_{s}^{\xi} \in C_{s}^{g} \text { for all } s \in[0, T]\right\} .
$$

Furthermore, the driver function $g$ satisfies for $(t, z) \in[0, T] \times \mathcal{L}^{2}(v)$

$$
g(t, z)=\sup _{h \in C_{t}^{g}} \int_{\mathbb{R}^{k} \backslash\{0\}} z(x) h(x) v(\mathrm{~d} x) .
$$

The proof follows by an adaptation of the arguments given in [36, Theorem A.25] and is omitted.

Remark 2.9 (i) Note that two driver functions $g_{1}$ and $g_{2}$ are equal if and only if the corresponding sets $C^{g_{1}}$ and $C^{g_{2}}$ in the representation (2.11) are equal.

(ii) Let $\bar{C}$ be a $\mathcal{U}$-measurable subset of $\mathcal{L}^{2}(v)$. If $C_{t}^{g}=\bar{C}$ for all $t \in[0, T]$, then the corresponding driver function is given by $g(t, z)=\bar{g}(z)$, where

$$
\bar{g}(z)=\sup _{\ell \in \bar{C}} \int_{\mathbb{R}^{k} \backslash\{0\}} z(x) \ell(x) v(\mathrm{~d} x), \quad z \in \mathcal{L}^{2}(v) .
$$

\subsection{Convergence}

We next turn to the question of convergence of a sequence $\left(\rho^{g^{(\pi)}},(\pi)\right)_{\pi}$ of dynamic coherent risk measures as in Definition 2.4 when the mesh size $\Delta=\Delta_{\pi}$ tends to zero. Let us suppose that $\left(\rho^{g^{(\pi)},(\pi)}\right)_{\pi}$ are driven by the random walks $\left(L^{(\pi)}\right)_{\pi}$ that are defined as

$$
\Delta L_{t}^{(\pi)}=J_{t} \sqrt{\Delta}, \quad J_{t} \stackrel{\text { i.i.d. }}{\sim}\left(p_{j}^{\Delta}, j \in \mathbb{Z}^{k}\right), \quad t \in \pi \backslash\{T\},
$$

for some probability distribution $\left(p_{j}^{\Delta}, j \in \mathbb{Z}^{k}\right)$ on $\mathbb{Z}^{k}$ that is given, in terms of a constant $c \geq 1$ (to be specified shortly) and a partition $\left(B_{j}^{\Delta}, j=\left(j_{1}, \ldots, j_{k}\right) \in \mathbb{Z}^{k}\right)$ of $(\sqrt{\Delta} \mathbb{Z})^{k}$ into block sets of the form

$$
B_{j}^{\Delta}=\prod_{j_{i}} A_{j_{i}}^{\Delta},
$$

where $A_{k}^{\Delta}=[k \sqrt{\Delta},(k+1) \sqrt{\Delta})$ if $k>0, A_{k}^{\Delta}=((k-1) \sqrt{\Delta}, k \sqrt{\Delta}]$ if $k<0$ and $A_{0}^{\Delta}=(-\sqrt{\Delta}, \sqrt{\Delta})$, by

$$
\begin{aligned}
& p_{j}^{\Delta}=v\left(B_{j}^{\Delta}\right) \Delta, \quad j \in \mathbb{Z}^{k} \backslash C_{\Delta}, \\
& p_{j}^{\Delta}=0, \quad j \in C_{\Delta} \backslash\{0\}, \\
& p_{0}^{\Delta}=1-\sum_{j \neq 0} p_{j}^{\Delta},
\end{aligned}
$$

where

$$
C_{\Delta}=\left\{j \in \mathbb{Z}^{k}:|j| \leq \sqrt{c_{\Delta} \nu_{2}}\right\}, \quad c_{\Delta}=c+(\log \Delta)^{-},
$$

where, as before, $v_{2}=\int_{\mathbb{R}^{k} \backslash\{0\}}|x|^{2} v(\mathrm{~d} x)$. 
When $\Delta \searrow 0$, we have by the dominated convergence theorem that

$$
\mathbb{E}\left[\left(L_{T}^{(\pi), r}+L_{T}^{(\pi), s}\right)^{2}\right] \longrightarrow T \int_{\mathbb{R}^{k} \backslash\{0\}}\left(x_{r}+x_{s}\right)^{2} v(\mathrm{~d} x), \quad r, s \in\{1, \ldots, k\},
$$

where $L_{T}^{(\pi), m}$ and $x_{m}, m \in\{1, \ldots, k\}$, denote the $m$ th coordinates of $L_{T}^{(\pi)}$ and $x \in \mathbb{R}^{k}$.

Moreover, we have by functional weak convergence theory (see e.g. [29, Theorem VII.3.7])

$$
L^{(\pi)} \stackrel{\mathrm{d}}{\longrightarrow} L \text { as } \Delta \searrow 0
$$

where $\stackrel{\mathrm{d}}{\longrightarrow}$ denotes convergence in law in the Skorokhod $J_{1}$-topology on the space $\mathbb{D}\left([0, T], \mathbb{R}^{k}\right)$ of $\mathbb{R}^{k}$-valued RCLL functions.

On a suitably chosen probability space, $L_{T}^{(\pi)}$ converges to $L_{T}$ almost surely as $\Delta \searrow 0$. The latter convergence also holds in a stronger sense thanks to moment conditions satisfied by $L_{T}^{(\pi)}$ that we show next. We define the value of $c$ in terms of $\varepsilon_{0}>0$ given in Assumption 2.5 by

$$
c=\sup _{x, y \in \mathbb{R}^{k}} \frac{|x+y|^{2+\varepsilon_{0}} \vee 1}{\left(|x|^{2+\varepsilon_{0}} \vee 1\right)\left(|y|^{2+\varepsilon_{0}} \vee 1\right)} .
$$

Lemma 2.10 The collection $\left(L^{(\pi)}\right)_{\pi}$ of random walks defined in (2.12)-(2.15) is such that for any uniform partition $\pi$ and $t \in \pi \backslash\{T\}$, we have $\mathbb{E}\left[\left|\Delta L_{t}^{(\pi)}\right|\right] / \sqrt{\Delta} \rightarrow 0$ as $\Delta \searrow 0$ and

$$
\mathbb{E}\left[\left|\Delta L_{t}^{(\pi)}\right|^{2+\varepsilon_{0}}\right] \leq \nu_{2+\varepsilon_{0}} \Delta, \quad \mathbb{P}\left[\left|\Delta L_{t}^{(\pi)}\right|=0\right] \geq 1-\frac{1}{c_{\Delta}},
$$

where $\varepsilon_{0}>0$ and $\nu_{2+\varepsilon_{0}}$ are as in Assumption 2.5 and $c_{\Delta}$ is given in (2.16) and (2.17). Furthermore, we have

$$
\sup _{\pi: \Delta_{\pi} \in \mathbb{R}_{+} \backslash\{0\}} \mathbb{E}\left[\left|L_{T}^{(\pi)}\right|^{2+\varepsilon_{0}}\right] \in \mathbb{R}_{+}
$$

Remark 2.11 Under the bound on the right-hand side of (2.18), we have numerical stability of the solutions to a sequence of BS $\Delta$ Es driven by $\left(L^{(\pi)}\right)$ (see [37, Theorem 3.4]).

Proof of Lemma 2.10 Letting $\pi=\pi_{\Delta}$ denote the partition with mesh size $\Delta \in \mathbb{R}_{+} \backslash\{0\}$ and $\varepsilon=\varepsilon_{0}$, a first observation is that for any $t \in \pi \backslash\{T\}, a \in \mathbb{R}_{+} \backslash\{0\}$ and $p \in[2,2+\varepsilon]$, we have by Chebyshev's inequality

$$
\begin{aligned}
\mathbb{P}\left[\left|\Delta L_{t}^{(\pi)}\right|>a\right] & \leq v\left(\left\{z \in \mathbb{R}^{k}:|z| \geq a\right\}\right) \Delta \\
& \leq \frac{v_{p}}{a^{p}} \Delta,
\end{aligned}
$$


where, as before, $v_{p}=\int_{\mathbb{R}^{k} \backslash\{0\}}|x|^{p} v(\mathrm{~d} x)$. By multiplying (2.20) by $p a^{p-1}$ and integrating, we have the estimate

$$
\mathbb{E}\left[\left|\Delta L_{t}^{(\pi)}\right|^{p}\right] \leq v_{p} \Delta, \quad p \in[2,2+\varepsilon] .
$$

Taking in (2.21) $p=2$ and $a=b \sqrt{c_{\Delta} \nu_{2} \Delta}$ and setting $b=1$ shows that

$$
\mathbb{P}\left[\left|\Delta L_{t}^{(\pi)}\right|>0\right]=\mathbb{P}\left[\left|\Delta L_{t}^{(\pi)}\right|>\sqrt{c_{\Delta} \nu_{2} \Delta}\right] \leq c_{\Delta}^{-1}
$$

which yields the bound on the right-hand side of (2.18), while integrating over $b \geq 1$ shows that $\mathbb{E}\left[\left|\Delta L_{t}^{(\pi)}\right|\right] / \sqrt{\Delta} \leq \sqrt{\nu_{2} / c_{\Delta}}$, which tends to zero as $\Delta \searrow 0$ in view of the form of $c_{\Delta}$.

To establish (2.19), the proof next proceeds analogously as that of the moment result for Lévy processes (see [44, Theorem 25.3]). The key step to transfer the uniform estimate of moments of the increments to a uniform estimate of moments of the random walk at $T$ is the estimate for submultiplicative functions $g$ (a function $g: \mathbb{R}^{k} \rightarrow \mathbb{R}$ is called submultiplicative if for some $b_{g} \in \mathbb{R}_{+}$and any $x, y \in \mathbb{R}^{k}$, we have $\left.g(x+y) \leq b_{g} g(x) g(y)\right)$ given by

$$
\mathbb{E}\left[g\left(L_{T}^{(\pi)}\right)\right]=\mathbb{E}\left[g\left(\sum_{t \in \pi \backslash\{T\}} \Delta L_{t}^{(\pi)}\right)\right] \leq b_{g}^{N-1} \mathbb{E}\left[g\left(\Delta L_{t_{1}}^{(\pi)}\right)\right]^{N},
$$

where we used that the increments $\Delta L_{t}^{(\pi)}, t \in \pi \backslash\{T\}$, are independent. For any $a \in \mathbb{R}_{+}$, the function $g_{a}(x):=|x|^{2+\varepsilon} \vee a$ is submultiplicative; see [44, Proposition 25.4]. From (2.22) and (2.23), we have that $\mathbb{E}\left[g_{1}\left(\Delta L_{t}^{(\pi)}\right)\right]$ is bounded above by

$$
\mathbb{E}\left[g_{0}\left(\Delta L_{t}^{(\pi)}\right)\right]+\mathbb{P}\left[\left|\Delta L_{t}^{(\pi)}\right| \in(0,1]\right] \leq v_{2+\varepsilon} \Delta+c_{\Delta}^{-1} .
$$

Combining the bounds (2.24) and (2.25) with the facts that $c$ defined in (2.17) is such that $b_{g_{1}}=c$ and $c \leq c_{\Delta}$, we have for all $N \in \mathbb{N}$ that

$$
\mathbb{E}\left[g_{1}\left(L_{T}^{(\pi)}\right)\right] \leq c^{N-1}\left(\frac{1}{c}+v_{2+\varepsilon} \Delta\right)^{N}=\frac{1}{c}\left(1+\frac{c v_{2+\varepsilon} T}{N}\right)^{N} .
$$

As the right-hand side of (2.26) is bounded above by $c^{-1} \exp \left(c v_{2+\varepsilon} T\right)$, we have (2.19) and the proof is complete.

The moment conditions in Lemma 2.10 carry over to those of path-functionals as follows.

Corollary 2.12 Assume that $F: \mathbb{D}\left([0, T], \mathbb{R}^{k}\right) \rightarrow \mathbb{R}$ satisfies, for some $c \in \mathbb{R}_{+}$,

$$
|F(\omega)| \leq c\|\omega\|_{\infty} \quad \text { for all } \omega \in \mathbb{D}\left([0, T], \mathbb{R}^{k}\right),
$$

where $\|\omega\|_{\infty}=\sup _{t \in[0, T]}|\omega(t)|$. Then, uniformly over partitions $\pi=\pi_{\Delta}$,

$$
\sup _{\Delta \in \mathbb{R}_{+} \backslash\{0\}} \mathbb{E}\left[\left|F\left(L^{(\pi)}\right)\right|^{2+\varepsilon_{0}}\right] \in \mathbb{R}_{+} .
$$


Proof For any partition $\pi$, an application of Doob's inequality to the centred random walk $\bar{L}_{t}^{(\pi)}=L_{t}^{(\pi)}-t \mathbb{E}\left[L_{1}^{(\pi)}\right]$ shows that

$$
\mathbb{E}\left[\sup _{t \in \pi}\left|\bar{L}_{t}^{(\pi)}\right|^{2+\varepsilon_{0}}\right] \leq \mathrm{const} \mathbb{E}\left[\left|\bar{L}_{T}^{(\pi)}\right|^{2+\varepsilon_{0}}\right] .
$$

The assertion follows by combining the estimate (2.28) with (2.27), the triangle inequality, the convexity of $x \mapsto|x|^{2+\varepsilon_{0}}$ and (2.19) in Lemma 2.10.

To guarantee that the convergence of the random walks $\left(L^{(\pi)}\right)_{\pi}$ carries over to the convergence of the corresponding BS $\triangle \mathrm{Es}$, we impose the following condition on the sequence of coherent driver functions $\left(g^{(\pi)}\right)_{\pi}$ and their piecewise constant RCLL interpolations $\left(\tilde{g}^{(\pi)}\right)_{\pi}$.

Condition 2.13 (i) The collection of functions $\left(g^{(\pi)}\right)_{\pi}$ is uniformly Lipschitzcontinuous with Lipschitz constants $K^{(\pi)}$ such that $\sup _{\pi} K^{(\pi)} \in \mathbb{R}_{+}$.

(ii) For any continuous function $h$ for which $\sup _{x \in \mathbb{R}^{k} \backslash\{0\}}|h(x)| /|x|$ is bounded and any $t \in[0, T]$, we have

$$
\lim _{\Delta \rightarrow 0} \tilde{g}^{(\pi)}(t, h)=g(t, h)
$$

The convergence result for BS $\Delta$ Es [37, Theorem 4.1] is phrased as follows in the current setting.

Theorem 2.14 Let $g$ be a coherent driver function, let $\left(L^{(\pi)}\right)_{\pi}$ be as in (2.12)-(2.15) and suppose that the sequence of coherent driver functions $\left(g^{(\pi)}\right)_{\pi}$ satisfies Condition 2.13. If $X^{(\pi)} \in \mathcal{L}^{2}\left(\mathcal{F}_{T}^{(\pi)}\right)$ and $X \in \mathcal{L}^{2}$ are such that $X^{(\pi)} \rightarrow X$ in distribution and the collection $\left(\left(X^{(\pi)}\right)^{2}\right)_{\pi}$ is uniformly integrable, then we have (with $\tilde{\rho}^{g^{(\pi)}},(\pi)$ the piecewise constant RCLL interpolation of $\rho^{g^{(\pi)},(\pi)}$ )

$$
\tilde{\rho}^{g^{(\pi)},(\pi)}\left(X^{(\pi)}\right) \stackrel{\mathrm{d}}{\longrightarrow} \rho^{g}(X) \text { as } \Delta \searrow 0 \text {. }
$$

\section{Choquet-type integrals and iterated versions}

\subsection{Choquet-type integrals}

We describe next the Choquet-type integrals that feature in the definition of dynamic spectral risk measures given in the next section. We refer to [18] for a treatment of the theory of nonlinear integration. The Choquet-type integrals we consider are given in terms of measure distortions that we define next.

Definition 3.1 Let $(\mathbb{U}, \mathcal{U}, \mu)$ be a measure space.

(i) $\Gamma:[0, \mu(\mathbb{U})] \rightarrow[0, \infty]$ is called a measure distortion if $\Gamma$ is continuous and increasing with $\Gamma(0)=0$. If $\Gamma(1)=1$, then $\Gamma$ is called a probability distortion. 
(ii) $\Gamma \circ \mu: \mathcal{U} \rightarrow[0, \infty]$ denotes the set function given by $(\Gamma \circ \mu)(A):=\Gamma(\mu(A))$ for $A \in \mathcal{U}$.

On a given measure space $(\mathbb{U}, \mathcal{U}, \mu)$, a set $A \in \mathcal{U}$ with $\mu(A)>0$ is called an atom if $C \subseteq A$ implies $\mu(C) \in\{0, \mu(A)\}$. We assume throughout that the measure distortions and associated measure spaces are of the following type:

Assumption 3.2 The measure $\mu$ on $(\mathbb{U}, \mathcal{U})$ is sigma-finite and has no atoms, and the measure distortion $\Gamma:[0, \mu(\mathbb{U})) \rightarrow \mathbb{R}_{+}$is bounded and such that

$$
K_{\Gamma}:=\int_{(0, \mu(\mathbb{U}))} \frac{\Gamma(y)}{2 y \sqrt{y}} \mathrm{~d} y \in \mathbb{R}_{+} .
$$

The Choquet-type integrals that we consider are defined as follows.

Definition 3.3 Let $(\mathbb{U}, \mathcal{U}, \mu)$ be a measure space and $\Gamma_{+}$and $\Gamma_{-}$associated measure distortions which satisfy Assumption 3.2.

(i) The Choquet-type integral $\mathrm{C}_{+}^{\Gamma_{+} \circ \mu}: \mathcal{L}_{+}^{2}(\mathbb{U}, \mathcal{U}, \mu) \rightarrow \mathbb{R}_{+}$is given by

$$
\mathrm{C}_{+}^{\Gamma_{+} \circ \mu}(f):=\int_{[0, \infty)}\left(\Gamma_{+} \circ \mu\right)(f>x) \mathrm{d} x, \quad f \in \mathcal{L}_{+}^{2}(\mathbb{U}, \mathcal{U}, \mu),
$$

where $\{f>x\}=\{z \in \mathbb{U}: f(z)>x\}$.

(ii) The Choquet-type integral $C^{\Gamma_{+} \circ \mu, \Gamma_{-} \circ \mu}: \mathcal{L}^{2}(\mathbb{U}, \mathcal{U}, \mu) \rightarrow \mathbb{R}$ is given by

$$
\mathrm{C}^{\Gamma_{+} \circ \mu, \Gamma_{-} \circ \mu}(f)=\mathrm{C}_{+}^{\Gamma_{+} \circ \mu}\left(f^{+}\right)-\mathrm{C}_{+}^{\Gamma_{-} \circ \mu}\left(f^{-}\right) .
$$

Remark 3.4 (i) To see that $\mathrm{C}_{+}^{\Gamma \circ \mu}(f) \in \mathbb{R}_{+}$for $f \in \mathcal{L}_{+}^{2}(\mu)$ and $\mu$ and $\Gamma$ satisfying Assumption 3.2, we note that by Chebyshev's inequality, monotonicity of $\Gamma$ and a change of variables, we have

$$
C_{+}^{\Gamma \circ \mu}(f)=\int_{0}^{\infty} \Gamma(\mu(f>x)) \mathrm{d} x \leq \int_{0}^{\infty} \Gamma\left(|f|_{2, \mu}^{2} / x^{2}\right) \mathrm{d} x=K_{\Gamma}|f|_{2, \mu}
$$

if $\mu(\mathbb{U})=\infty$. If $\mu(\mathbb{U})<\infty$, a similar line of reasoning gives $\mathrm{C}_{+}^{\Gamma \circ \mu}(f) \leq K_{\Gamma}^{\prime}\|f\|_{2, \mu}$ with $K_{\Gamma}^{\prime}=K_{\Gamma}+\Gamma(\mu(\mathbb{U})) / \sqrt{\mu(\mathbb{U})}$.

(ii) Taking in Definition $3.3(\mathbb{U}, \mathcal{U}, \mu)=\left(\Omega, \mathcal{F}_{T}, \mathbb{P}\right)$, and taking the measure distortions $\Gamma_{+}$and $\Gamma_{-}$equal to a continuous probability distortion $\Psi$ and the function $\widehat{\Psi}$ given by $\widehat{\Psi}(x)=1-\Psi(1-x)$ for $x \in[0,1]$, it is straightforward to check that $\Psi \circ \mathbb{P}$ is a capacity and the Choquet-type integral of $X \in \mathcal{L}^{2}$ in (3.2) coincides with the classical Choquet expectation corresponding to $\Psi \circ \mathbb{P}$, i.e.,

$$
C^{\Psi \circ \mathbb{P}, \widehat{\Psi} \circ \mathbb{P}}(X)=\int_{0}^{\infty}(\Psi \circ \mathbb{P})(X>x) \mathrm{d} x-\int_{-\infty}^{0}(1-(\Psi \circ \mathbb{P})(X>x)) \mathrm{d} x .
$$

Moreover, as we have $\widehat{\Psi}(x) \leq x \leq \Psi(x)$ for $x \in[0,1]$, it follows that

$$
\mathrm{C}^{\Psi \circ \mathbb{P}, \widehat{\Psi} \circ \mathbb{P}}(X) \geq \mathbb{E}[X],
$$


and we have equality in (3.3) for all $X \in \mathcal{L}^{2}$ if and only if $\Psi(x)=\widehat{\Psi}(x)=x$ for $x \in[0,1]$.

We record next a robust representation result for Choquet-type integrals that plays an important role in the sequel. Let $\mathcal{M}_{p, \mu}, p \geq 1$, denote the set of measures $m$ on $(\mathbb{U}, \mathcal{U})$ that are absolutely continuous with respect to a given measure $\mu$ on this space with Radon-Nikodým derivatives such that $\frac{\mathrm{d} m}{\mathrm{~d} \mu} \in \mathcal{L}_{+}^{p}(\mu)$.

Proposition 3.5 For a given concave measure distortion $\Gamma$ and measure $\mu$ on $(\mathbb{U}, \mathcal{U})$ satisfying Assumption 3.2, define

$$
\mathcal{M}_{1, \mu}^{\Gamma}:=\left\{m \in \mathcal{M}_{1, \mu}^{a c}: m(A) \leq \Gamma(\mu(A)) \text { for all } A \in \mathcal{U} \text { with } \mu(A)<\infty\right\} .
$$

Then $\mathrm{C}_{+}^{\Gamma \circ \mu}: \mathcal{L}_{+}^{2}(\mu) \rightarrow \mathbb{R}_{+}$is $K_{\Gamma^{-} \text {-Lipschitz-continuous and }}$

$$
\mathrm{C}_{+}^{\Gamma \circ \mu}(f)=\sup \left\{m(f): m \in \mathcal{M}_{1, \mu}^{\Gamma}\right\} \quad \text { for } f \in \mathcal{L}_{+}^{2}(\mu) .
$$

Furthermore, the subgradients of $\mathrm{C}_{+}^{\Gamma \circ \mu}$ (i.e., the elements of the dual set $\mathcal{M}_{1, \mu}^{\Gamma}$ ) are

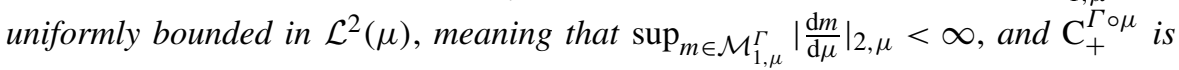
positively homogeneous and subadditive, that is, for any $\lambda \in \mathbb{R}_{+}$and $f, g \in \mathcal{L}_{+}^{2}(\mu)$,

$$
\mathrm{C}_{+}^{\Gamma \circ \mu}(\lambda f)=\lambda \mathrm{C}_{+}^{\Gamma \circ \mu}(f), \quad \mathrm{C}_{+}^{\Gamma \circ \mu}(f+g) \leq \mathrm{C}_{+}^{\Gamma \circ \mu}(f)+\mathrm{C}_{+}^{\Gamma \circ \mu}(g) .
$$

Proof The representation in (3.4) is known to hold true when (a) $\Gamma$ (1) $=1$ and (b) $\mu$ has unit mass and (c) $\mathcal{M}_{1, \mu}^{\Gamma}$ is replaced by the set of $m \in \mathcal{M}_{1, \mu}^{\Gamma}$ with $m(\mathbb{U})=1$ (see [8] and [26, Corollary 4.80]). We note that by positive homogeneity and (a) and (b), (c) is not needed for the representation in (3.4) to hold true. Let $\varepsilon>0$, let $\mu$ be as given, let $m \in \mathcal{M}_{1, \mu}^{\Gamma}$, and denote by $O_{\varepsilon}, \varepsilon>0$, a collection of sets with $0<\mu\left(O_{\varepsilon}\right)<\infty$ and such that $O_{\varepsilon} \nearrow \mathbb{U}$. Denoting

$$
\begin{aligned}
c_{\varepsilon} & :=\mu\left(O_{\varepsilon}\right), & \Gamma_{\varepsilon}(\cdot) & :=\Gamma\left(c_{\varepsilon} \cdot\right), \\
m_{\varepsilon}(\mathrm{d} x) & :=I_{O_{\varepsilon}}(|x|) m(\mathrm{~d} x), & \mu_{\varepsilon}(\mathrm{d} x) & :=c_{\varepsilon}^{-1} I_{O_{\varepsilon}}(|x|) \mu(\mathrm{d} x),
\end{aligned}
$$

we thus have for any $f \in \mathcal{L}_{+}^{2}(\mu)$ that

$$
\begin{aligned}
\frac{1}{\Gamma_{\varepsilon}(1)} C_{+}^{\Gamma_{\varepsilon} \circ \mu_{\varepsilon}}(f) & =\sup \left\{m(f): m \in \mathcal{M}_{1, \mu_{\varepsilon}}^{\Gamma_{\varepsilon} / \Gamma_{\varepsilon}(1)}\right\} \\
& =\sup \left\{\frac{1}{\Gamma_{\varepsilon}(1)} m_{\varepsilon}(f): m \in \mathcal{M}_{1, \mu}^{\Gamma}\right\} .
\end{aligned}
$$

Since one can readily verify by an application of the monotone convergence theorem that $\mathrm{C}_{+}^{\Gamma_{\varepsilon} \circ \mu_{\varepsilon}}(f) \nearrow \mathrm{C}_{+}^{\Gamma \circ \mu}(f)$ and $m_{\varepsilon}(f) \nearrow m(f)$ as $\varepsilon \searrow 0$, and since $\Gamma_{\varepsilon}(1) \in \mathbb{R}_{+} \backslash\{0\}$, we obtain (3.4) by taking $\varepsilon \searrow 0$ in (3.6).

The positive homogeneity and convexity of $\mathrm{C}_{+}^{\Gamma \circ \mu}(f)$ as stated in (3.5) follow as direct consequences of the robust representation in (3.4). 
Next we turn to the proof of Lipschitz-continuity. We observe that the robust representation (3.4) of $\mathrm{C}_{+}^{\Gamma \circ \mu}$ implies that for $u, v \in \mathcal{L}_{+}^{2}(\mu)$,

$$
\left|\mathrm{C}_{+}^{\Gamma \circ \mu}(u)-\mathrm{C}_{+}^{\Gamma \circ \mu}(v)\right| \leq\left|\mathrm{C}_{+}^{\Gamma \circ \mu}(v-u)\right| \vee\left|\mathrm{C}_{+}^{\Gamma \circ \mu}(u-v)\right| .
$$

Using a similar estimate as in Remark 3.4(i), we note that for $m \in \mathcal{M}_{1, \mu}^{\Gamma}$ and some $c \in \mathbb{R}_{+}$,

$$
\begin{aligned}
\left|\frac{\mathrm{d} m}{\mathrm{~d} \mu}\right|_{2, \mu}^{2} & =\int\left(\frac{\mathrm{d} m}{\mathrm{~d} \mu}\right)^{2} \mathrm{~d} \mu=\int \frac{\mathrm{d} m}{\mathrm{~d} \mu} \mathrm{d} m=\int_{0}^{\infty} m\left(\frac{\mathrm{d} m}{\mathrm{~d} \mu}>x\right) \mathrm{d} x \\
& \leq \int_{0}^{\infty} \Gamma\left(\mu\left(\frac{\mathrm{d} m}{\mathrm{~d} \mu}>x\right)\right) \mathrm{d} x \leq c\left|\frac{\mathrm{d} m}{\mathrm{~d} \mu}\right|_{2, \mu},
\end{aligned}
$$

which implies $\sup _{m \in \mathcal{M}_{1, \mu}^{\Gamma}}\left|\frac{\mathrm{d} m}{\mathrm{~d} \mu}\right|_{2, \mu} \leq c$, and hence we obtain for $u \in \mathcal{L}_{+}^{2}(\mu)$ that $\left|\mathrm{C}_{+}^{\Gamma \circ \mu}(u)\right| \leq c|u|_{2, \mu}$ by (3.4). The latter bound together with (3.7) yields the stated Lipschitz-continuity.

\subsection{Conditional and iterated Choquet integrals}

Analogously, we define $\mathcal{F}_{t}$-conditional Choquet-type integrals as follows.

Definition 3.6 For any $t \in[0, T]$ and probability distortions $\Psi$ and $\bar{\Psi}$ satisfying Assumption 3.2 (relative to the measure $\mathbb{P}$ restricted to $\left(\Omega, \mathcal{F}_{t}\right)$ ), the conditional Choquet-type integral $\mathrm{C}^{\Psi \circ \mathbb{P}, \bar{\Psi} \circ \mathbb{P}}\left(\cdot \mid \mathcal{F}_{t}\right): \mathcal{L}^{2} \rightarrow \mathcal{L}_{t}^{2}$ is given by, for $X \in \mathcal{L}^{2}$,

$$
C^{\Psi \circ \mathbb{P}, \bar{\Psi} \circ \mathbb{P}}\left(X \mid \mathcal{F}_{t}\right):=\int_{\mathbb{R}_{+}} \Psi\left(\mathbb{P}\left[X^{+}>x \mid \mathcal{F}_{t}\right]\right) \mathrm{d} x-\int_{\mathbb{R}_{+}} \bar{\Psi}\left(\mathbb{P}\left[X^{-}>x \mid \mathcal{F}_{t}\right]\right) \mathrm{d} x .
$$

Remark 3.7 (i) Reasoning similarly as in Remark 3.4 (i) and as in the proof of Lemma 3.5, we have that for any $X \in \mathcal{L}^{2}, \mathrm{C}^{\Psi \circ \mathbb{P}, \bar{\Psi} \circ \mathbb{P}}\left(X \mid \mathcal{F}_{t}\right)$ is square-integrable, and the map $C^{\Psi \circ \mathbb{P}, \bar{\Psi} \circ \mathbb{P}}\left(\cdot \mid \mathcal{F}_{t}\right)$ is Lipschitz-continuous on $\mathcal{L}^{2}$ with Lipschitz constant $K_{\Psi}+K_{\bar{\Psi}}$ (which are given by the constant $K_{\Gamma}$ in (3.1) with $\mu(\mathbb{U})=1$ and $\Gamma$ equal to $\Psi$ and $\bar{\Psi}$, respectively).

(ii) The conditional Choquet expectation in (3.2) of $X \in \mathcal{L}^{2}$ with $\bar{\Psi}=\widehat{\Psi}$ may equivalently be expressed as weighted integral of the conditional expected shortfall of $X$ at different levels. Specifically, associated to any concave probability distortion $\Psi$ is a unique Borel measure $\mu$ on $[0,1]$ defined by $\mu(\{0\})=0$ and $\mu(\mathrm{d} s)=s F(\mathrm{~d} s)$ for $s \in(0,1]$, where $F$ is the locally finite positive measure given in terms of the right derivative $\Psi_{+}^{\prime}$ of $\Psi$ by $F((s, 1])=\Psi_{+}^{\prime}(s)$ (see [26, Theorem 4.70]). It is straightforward to check that $\Psi$ satisfies Assumption 3.2 if and only if

$$
\int_{(0,1]} \frac{1}{\sqrt{s}} \mu(\mathrm{d} s) \in \mathbb{R}_{+} \backslash\{0\} .
$$


The conditional Choquet expectation in Definition 3.6 can then be expressed in terms of the measure $\mu$ and the $\mathcal{F}_{t}$-conditional expected shortfall as

$$
\mathrm{C}^{\Psi \circ \mathbb{P}, \widehat{\Psi} \circ \mathbb{P}}\left(X \mid \mathcal{F}_{t}\right)=\int_{(0,1]} \mathrm{ES}_{\lambda}\left(-X \mid \mathcal{F}_{t}\right) \mu(\mathrm{d} \lambda), \quad X \in \mathcal{L}^{2},
$$

where the $\mathcal{F}_{t}$-conditional expected shortfall $\operatorname{ES}_{\lambda}\left(X \mid \mathcal{F}_{t}\right)$ of $X \in \mathcal{L}^{2}$ at level $\lambda \in(0,1]$ is given in terms of the $\mathcal{F}_{t}$-conditional value-at-risk

$$
\operatorname{VaR}_{\lambda}\left(X \mid \mathcal{F}_{t}\right)=\inf \left\{z \in \mathbb{R}: \mathbb{P}\left[X<-z \mid \mathcal{F}_{t}\right]<\lambda\right\}
$$

at level $\lambda$ by

$$
\operatorname{ES}_{\lambda}\left(X \mid \mathcal{F}_{t}\right)=\frac{1}{\lambda} \int_{0}^{\lambda} \operatorname{VaR}_{u}\left(X \mid \mathcal{F}_{t}\right) \mathrm{d} u, \quad \lambda \in(0,1] .
$$

The proof of (3.8) follows by a straightforward adaptation to the conditional setting of the proof for the static setting given in Föllmer and Schied [26, Corollary 4.77].

(iii) It follows from the representation in (3.8) that the collection of conditional Choquet expectations $X \mapsto \mathrm{C}^{\Psi \circ \mathbb{P}, \bar{\Psi} \circ \mathbb{P}}\left(-X \mid \mathcal{F}_{t}\right), t \in[0, T], X \in \mathcal{L}^{2}$, is a dynamic coherent risk measure in the sense of Definition 2.1 (with $\mathcal{I}=[0, T]$ ).

One way to define a sequence of conditional spectral risk measures adapted to the filtration $\mathbf{F}^{(\pi)}=\left(\mathcal{F}_{t}^{(\pi)}\right)_{t \in \pi}$ is recursive in terms of conditional Choquet-integrals, as follows.

Definition 3.8 Given a concave probability distortion $\Psi$ satisfying Assumption 3.2 and a filtration $\mathbf{F}^{(\pi)}=\left(\mathcal{F}_{t}^{(\pi)}\right)_{t \in \pi}$, the corresponding iterated spectral risk measure $\mathrm{S}=\left(\mathrm{S}_{t}\right)_{t \in \pi}, \mathrm{S}_{t}: \mathcal{L}^{2}\left(\mathcal{F}_{T}^{(\pi)}\right) \rightarrow \mathcal{L}^{2}\left(\mathcal{F}_{t}^{(\pi)}\right)$, is defined recursively on the grid $\pi=\pi_{\Delta}$ by

$$
\mathrm{S}_{t}(X)= \begin{cases}\mathrm{C}^{\Psi \circ \mathbb{P}, \widehat{\Psi} \circ \mathbb{P}}\left(\mathrm{S}_{t+1}(X) \mid \mathcal{F}_{t}^{(\pi)}\right), & t \in \pi \backslash\{T\} \\ -X, & t=T .\end{cases}
$$

The class of iterated spectral risk measures defined above contains in particular the iterated tail conditional expectation proposed in [28] and is closely related to the dynamic weighted $V @ R$ defined in [12] for adapted processes via its robust representation. As already noted in the proof of Proposition 3.5, in the static case such a representation was derived in [8] for bounded random variables; see also [26, Theorems 4.79 and 4.94], and see [11] for the extension to the set of measurable random variables (we refer to [22] for families of dynamic risk measures defined via stochastic distortion probabilities in a binomial tree setting; see [13] for a general theory of finite-state BSDEs).

We show next that iterated spectral risk measures are discrete-time time-consistent dynamic coherent risk measures, and we identify the driver function of the associated $\mathrm{BS} \Delta \mathrm{E}$. 
Proposition 3.9 The iterated spectral risk measure $\mathrm{S}=\left(\mathrm{S}_{t}\right)_{t \in \pi}$ in Definition 3.8 is a discrete-time coherent risk measure $\rho^{\bar{g}_{\Delta}, \pi}$ with driver function $\bar{g}_{\Delta}$ given by

$$
\bar{g}_{\Delta}(t, h)=\frac{1}{\Delta}\left(\mathrm{C}^{\Psi \circ\left(v^{(\pi)} \Delta\right), \widehat{\Psi} \circ\left(v^{(\pi)} \Delta\right)}\left(h(f) I_{\{f \neq 0\}}\right)-\Delta \int_{\mathbb{R}^{k} \backslash\{0\}} h(x) v^{(\pi)}(\mathrm{d} x)\right),
$$

where $v^{(\pi)}$ is defined in (2.1).

Proof It follows from Proposition 3.5 that the function $\bar{g}_{\Delta}$ defined in (3.9) is a coherent driver function in the sense of Definition 2.3 with $\mathcal{I}=\pi$ and $\mu=v^{(\pi)}$. Let $X \in \mathcal{L}^{2}\left(\mathcal{F}^{(\pi)}\right)$ be arbitrary and denote by $\left(Y^{(\pi)}, Z^{(\pi)}\right)$ the solution of the BS $\Delta \mathrm{E}$ with driver function $\bar{g}_{\Delta}$. To show that the dynamic coherent risk measure corresponding to $\bar{g}_{\Delta}$ coincides with the spectral risk measure $S=\left(S_{t}\right)_{t \in \pi}$, it suffices to verify that

$$
\bar{g}_{\Delta}\left(t, Z_{t}^{(\pi)}\right) \Delta=\mathrm{S}_{t}(X)-\mathbb{E}\left[\mathrm{S}_{t+1}(X) \mid \mathcal{F}_{t}^{(\pi)}\right] .
$$

Letting $t \in \pi \backslash\{T\}$ and denoting $\Delta L=\Delta L_{t}^{(\pi)}$, we note from Definition 3.8 and (2.3) that $\mathrm{S}_{t}(X)-\mathbb{E}\left[\mathrm{S}_{t+1}(X) \mid \mathcal{F}_{t}^{(\pi)}\right]$ is equal to

$$
\begin{aligned}
\mathrm{C}^{\Psi \circ \mathbb{P},}, \widehat{\Psi} \circ \mathbb{P} & \left(\mathrm{S}_{t+1}(X) \mid \mathcal{F}_{t}^{(\pi)}\right)-\mathbb{E}\left[\mathrm{S}_{t+1}(X) \mid \mathcal{F}_{t}^{(\pi)}\right] \\
= & \mathrm{C}^{\Psi \circ \mathbb{P}, \widehat{\Psi} \circ \mathbb{P}}\left(Z_{t}^{(\pi)}(\Delta L) I_{\{\Delta L \neq 0\}} \mid \mathcal{F}_{t}^{(\pi)}\right) \\
& -\mathbb{E}\left[Z_{t}^{(\pi)}(\Delta L) I_{\{\Delta L \neq 0\}} \mid \mathcal{F}_{t}^{(\pi)}\right] \\
= & \left.\left(\mathrm{C}^{\Psi \circ\left(v^{(\pi)} \Delta\right), \widehat{\Psi} \circ\left(v^{(\pi)} \Delta\right)}\left(h(f) I_{\{f \neq 0\}}\right)-\Delta \int_{\mathbb{R}^{k} \backslash\{0\}} h(x) v^{(\pi)}(\mathrm{d} x)\right)\right|_{h=Z_{t}^{(\pi)}},
\end{aligned}
$$

where we used that due to stationarity of the increments of $L^{(\pi)}, \Delta L_{t}^{(\pi)}$ (which has law $v^{(\pi)} \Delta$ ) is independent of $t$. Thus we have (3.10) and the proof is complete.

\section{Dynamic spectral risk measures}

With the previous results in hand, we move to the definition of dynamic spectral risk measures in continuous time. Let us fix in the sequel a pair of concave measure distortion functions $\Gamma_{+}$and $\Gamma_{-}$that satisfy Assumption 3.2 and are such that $\Gamma_{-}(x) \leq x$ for $x \in \mathbb{R}_{+}$. We define dynamic spectral risk measures to be those time-consistent dynamic coherent risk measures $\rho^{g}$ for which the driver functions $g$ are given in terms of Choquet integrals, as follows.

Definition 4.1 The spectral driver function $\bar{g}: \mathcal{L}^{2}(v) \rightarrow \mathbb{R}_{+}$is given by

$$
\bar{g}(u):=\mathrm{C}_{+}^{\Gamma_{+} \circ v}\left(u^{+}\right)+\mathrm{C}_{+}^{\Gamma_{-} \circ v}\left(u^{-}\right)
$$

for $u \in \mathcal{L}^{2}(v)$. 
By Lemma 3.5, we have that $\bar{g}$ is Lipschitz-continuous, positively homogeneous and convex, so that $\bar{g}$ is a coherent driver function in the sense of Definition 2.3. The corresponding time-consistent dynamic coherent risk measure $\rho^{\bar{g}}$ is the object of study for the remainder of the paper.

Definition 4.2 The dynamic coherent risk measure $\rho^{\bar{g}}$ with spectral driver function $\bar{g}$ given in Definition 4.1 is called the (continuous-time) dynamic spectral risk measure corresponding to the measure distortions $\Gamma_{+}$and $\Gamma_{-}$.

We next show that a dynamic spectral risk measure admits a dual representation of the form (2.9) and (2.10) with a representing set that is explicitly expressed in terms of the measure distortions $\Gamma_{+}$and $\Gamma_{-}$, as follows.

Theorem 4.3 Let $X \in \mathcal{L}^{2}, t \in[0, T]$ and let $\bar{g}$ be a spectral driver function. The dynamic spectral risk measure $\rho^{\bar{g}}$ satisfies the dual representation in (2.9) and (2.10) with representing set $C^{\bar{g}}$ given by

$$
C^{\bar{g}}=\left\{H \in \mathcal{L}^{2}(\nu): \begin{array}{c}
\text { for any } A \in \mathcal{B}\left(\mathbb{R}^{k} \backslash\{0\}\right) \text { with } v(A)<\infty, \\
-\Gamma_{-}(\nu(A)) \leq \int_{A} H \mathrm{~d} \nu \leq \Gamma_{+}(\nu(A))
\end{array}\right\},
$$

where $\int_{A} H \mathrm{~d} v=\int_{A} H(x) v(\mathrm{~d} x)$.

Example 4.4 The risk of a positive or negative jump arriving with a size larger than $a$, as quantified by the dynamic spectral risk measure $\rho^{\bar{g}}$, may be explicitly expressed in terms of $v, \Gamma_{+}$and $\Gamma_{-}$, as we show next. For any $a \in \mathbb{R}_{+} \backslash\{0\}$, let $I(a)=I_{\left\{\sup _{t \in[0, T]}\left|\Delta L_{t}\right| \leq a\right\}}=\left\{N_{T}^{a}=0\right\}, N_{T}^{a}=\#\left\{t \in[0, T]:\left|\Delta L_{t}\right|>a\right\}$ and $\bar{v}(a)=v(\{y:|y|>a\})$. While $\mathbb{E}[I(a)]=\exp (-\bar{v}(a) T)$ (since $N_{T}$ has a Poisson distribution with parameter $T \bar{v}(a))$, the values of $I(a)$ and $-I(a)$ under $\rho^{\bar{g}}$ are given by

$$
\begin{aligned}
\rho_{0}^{\bar{g}}(I(a)) & =-\exp \left(-T\left(\bar{v}(a)+\Gamma_{+}(\bar{v}(a))\right)\right), \\
\rho_{0}^{\bar{g}}(-I(a)) & =\exp \left(-T\left(\bar{v}(a)-\Gamma_{-}(\bar{v}(a))\right)\right) .
\end{aligned}
$$

These expressions follow by using the dual representation in Theorem 4.3 and Girsanov's theorem (see [29, Theorems III.3.24 and III.5.19]); indeed, we have that $\rho_{0}^{\bar{g}}(I(a))$ is equal to

$$
\begin{aligned}
\sup _{\mathbb{Q}^{\xi} \in \mathcal{S}^{g}} \mathbb{E}^{\mathbb{Q}^{\xi}}[-I(a)] & =\sup _{\mathbb{Q}^{\xi} \in \mathcal{S}^{g}} \mathbb{E}\left[-\exp \left(-\int_{0}^{T} \int_{\{|y|>a\}}\left(1+H_{t}^{\xi}(y)\right) v(\mathrm{~d} y) \mathrm{d} t\right)\right] \\
& =-\exp (-T \bar{v}(a)) \exp \left(-T \Gamma_{+}(\bar{v}(a))\right),
\end{aligned}
$$

while the expression for $\rho_{0}^{\bar{g}}(-I(a))$ follows in a similar manner. 
Proof of Theorem 4.3 In view of Theorem 2.8 and Remark 2.9, it suffices to verify that for any $h \in L^{2}(v)$, we have

$$
\sup _{\ell \in C^{\bar{g}}} \int h \ell \mathrm{d} v=\mathrm{C}_{+}^{\Gamma_{+} \circ v}\left(h^{+}\right)+\mathrm{C}_{+}^{\Gamma_{-} \circ v}\left(h^{-}\right),
$$

where $\int h \ell \mathrm{d} v=\int_{\mathbb{R}^{k} \backslash\{0\}} h(x) \ell(x) v(\mathrm{~d} x)$. Our next observation is that the set $C^{\bar{g}}$ in (4.1) admits the equivalent representation

$$
C^{\bar{g}}=\left\{U \in \mathcal{L}^{2}(v): \begin{array}{l}
\text { for any } A \in \mathcal{B}\left(\mathbb{R}^{\ell} \backslash\{0\}\right) \text { with } v(A)<\infty, \\
\int_{A} U^{+} \mathrm{d} v \leq \Gamma_{+}(v(A)), \int_{A} U^{-} \mathrm{d} v \leq \Gamma_{-}(v(A))
\end{array}\right\} .
$$

To see that this is the case, we note that for any $U \in L^{2}(v)$, we have $-U^{-} \leq U \leq U^{+}$, while $U^{+}=U_{1}$ and $-U^{-}=U_{2}$ for $U_{1}=U I_{\{U \geq 0\}}$ and $U_{2}=U I_{\{U<0\}}$.

To see that (4.2) holds, we note from (4.3), Proposition 3.5 and the identity

$$
h \ell^{*}=h^{+} \ell_{1}^{+}+h^{-} \ell_{2}^{-}, \quad \ell^{*}=\ell_{1}^{+} I_{\{h>0\}}-\ell_{2}^{-} I_{\{h<0\}},
$$

for any $h, \ell_{1}, \ell_{2} \in \mathcal{L}^{2}(v)$ that $\bar{g}(h)=\sup _{\ell \in C^{\bar{g}}} \int h \ell \mathrm{d} v$ is bounded below by

$$
\sup _{\ell_{1}, \ell_{2} \in C^{\bar{s}}} \int h \ell^{*} \mathrm{~d} v=\sup _{\ell \in C^{\bar{g}}} \int h^{+} \ell^{+} \mathrm{d} \nu+\sup _{\ell \in C^{\bar{g}}} \int h^{-} \ell^{-} \mathrm{d} \nu,
$$

which is by Proposition 3.5 equal to $\mathrm{C}_{+}^{\Gamma_{+} \text {ov }}\left(h^{+}\right)+\mathrm{C}_{+}^{\Gamma_{-} \text {ov }}\left(h^{-}\right)$. Given this lower bound and the fact that $\bar{g}(h)$ is bounded above by

$$
\sup _{\ell \in C^{\bar{s}}} \int h^{+} \ell \mathrm{d} v+\sup _{\ell \in C^{\bar{s}}} \int h^{-}(-\ell) \mathrm{d} v \leq \sup _{\ell \in C^{\bar{s}}} \int h^{+} \ell^{+} \mathrm{d} \nu+\sup _{\ell \in C^{\bar{g}}} \int h^{-} \ell^{-} \mathrm{d} \nu,
$$

we conclude that (4.2) holds true.

\section{Limit theorem}

We next turn to the functional limit theorem which shows that dynamic spectral risk measures arise as a limit of iterated spectral risk measures, under a suitable scaling of the corresponding probability distortions. We suppose that uniformly in $p \in[0,1]$, $\Psi_{\Delta}(p)-p$ scales in the mesh size $\Delta$ and the measure distortions $\Gamma_{+}$and $\Gamma_{-}$as

$$
\Psi_{\Delta}(p)=p+\Delta\left(\Gamma_{+}(p / \Delta) I_{\left[0, \frac{1}{2}\right]}(p)+\Gamma_{-}((1-p) / \Delta) I_{\left(\frac{1}{2}, 1\right]}(p)\right)+o(\Delta)(\Delta \searrow 0) .
$$

Specifically, the condition that we require is phrased as follows.

Definition 5.1 We denote by $\left(\Psi_{\Delta}\right)_{\Delta \in(0,1]}$ a sequence of probability distortions that is such that $\Psi_{\Delta}$ and $\widehat{\Psi}_{\Delta}$ given by $\widehat{\Psi}_{\Delta}(p)=1-\Psi_{\Delta}(1-p)$ satisfy Assumption 3.2 with respect to the measure $\mu(\mathrm{d} x):=\mathbb{P}\left[\Delta L_{t_{1}}^{(\pi)} \in \mathrm{d} x\right]$, and we have

$$
\lim _{\Delta \searrow 0} \Upsilon_{\Delta}=0, \quad \Upsilon_{\Delta}=\sup _{x \in(0,1)}\left|\frac{\Psi_{\Delta}(x)-x}{\Gamma_{\Delta}(x) \Delta}-1\right|,
$$


where for $\Delta \in(0,1]$ and $x \in[0,1]$,

$$
\Gamma_{\Delta}(x)=\Gamma_{+}(x / \Delta) I_{\left[0, \frac{1}{2}\right]}(x)+\Gamma_{-}((1-x) / \Delta) I_{\left(\frac{1}{2}, 1\right]}(x) .
$$

Here we recall that $\Gamma_{+}$and $\Gamma_{-}$denote the given concave measure distortions which are such that $\Gamma_{-}(x) \leq x$ for $x \in \mathbb{R}_{+}$and Assumption 3.2 holds with $\mu(\mathrm{d} x):=v(\mathrm{~d} x)$ and $\Gamma:=\Gamma_{+}$or $\Gamma_{-}$.

The functional limit result is phrased in terms of the sequence of piecewise constant RCLL extensions $\left(\tilde{L}^{(\pi)}\right)_{\pi}$ of the random walks $\left(L^{(\pi)}\right)_{\pi}$ given by

$$
\tilde{L}_{t}^{(\pi)}:=L_{\Delta^{-1}[t \Delta]}^{(\pi)}, \quad t \in[0, T],
$$

where $[r]=\sup \{n \in \mathbb{N} \cup\{0\}: n \leq r\}$ for $r \in \mathbb{R}_{+}$.

Theorem 5.2 Given a sequence of probability distortions $\left(\Psi_{\Delta}\right)_{\Delta \in(0,1]}$ as in Definition 5.1 and given filtrations $\mathbf{F}^{(\pi)}=\left(\mathcal{F}_{t}^{(\pi)}\right)_{t \in \pi}$, let $\mathrm{S}^{\Delta}=\left(\mathrm{S}_{t}^{\Delta}\right)_{t \in \pi}, \Delta \in(0,1]$, denote the corresponding iterated spectral risk measures as in Definition 3.8 and let $\bar{g}$ denote the spectral driver function from Definition 4.1. Let the set of $\omega \in \mathbb{D}\left([0, T], \mathbb{R}^{k}\right)$ at which $F: \mathbb{D}\left([0, T], \mathbb{R}^{k}\right) \rightarrow \mathbb{R}$ is discontinuous in the Skorokhod $J_{1}$-topology be a nullset under the law of $L$ and assume that for some $c \in \mathbb{R}_{+}$,

$$
|F(\omega)| \leq c\|\omega\|_{\infty} \quad \text { for all } \omega \in \mathbb{D}\left([0, T], \mathbb{R}^{k}\right) .
$$

Then we have

$$
\tilde{\mathrm{S}}^{\Delta}\left(F\left(\tilde{L}^{(\pi)}\right)\right) \stackrel{\mathrm{d}}{\longrightarrow} \rho^{\bar{g}}(F(L)), \quad \Delta \searrow 0
$$

where $\tilde{\mathrm{S}}_{t}^{\Delta}=\mathrm{S}_{\Delta^{-1}[t \Delta]}^{\Delta}, t \in[0, T]$.

Remark 5.3 (i) Given two concave probability distortions $\Psi_{+}$and $\Psi_{-}$satisfying the integrability condition (3.1) (with $\mu(\mathbb{U})=1$ ), one may explicitly construct a sequence $\left(\Psi_{\Delta}\right)_{\Delta \in(0,1]}$ satisfying Definition 5.1 via

$$
\Psi_{\Delta}(p)=p+\left(\Gamma_{+}(p / \Delta) I_{\left[0, \frac{1}{2}\right]}(p)+\Gamma_{-}((1-p) / \Delta) I_{\left(\frac{1}{2}, 1\right]}(p)\right) \Delta, \quad p \in[0,1],
$$

where inspired by [21], we suppose that the functions $\Gamma_{+}, \Gamma_{-}: \mathbb{R}_{+} \rightarrow \mathbb{R}_{+}$are given by

$$
\Gamma_{+}(x)=a \Psi_{+}\left(1-\mathrm{e}^{-c x}\right), \quad \Gamma_{-}(x)=\frac{b}{d} \Psi_{-}\left(1-\mathrm{e}^{-d x}\right), \quad x \in \mathbb{R}_{+},
$$

for some $a, b, c$ and $d \in \mathbb{R}_{+} \backslash\{0\}$ satisfying the restrictions

$$
\Gamma_{+}(1 /(2 \Delta))=\Gamma_{-}(1 /(2 \Delta))<1 /(2 \Delta), \quad b \Psi_{-}^{\prime}(0+) \in(0,1),
$$

where $f^{\prime}(0+)$ denotes the right derivative of a function $f$ at $x=0$. It is straightforward to check that for any $\Delta \in(0,1], \Psi_{\Delta}$ is a concave probability distortion (the 
first condition in (5.3) guarantees continuity at $p=1 / 2$ and $\left.\Psi_{\Delta}(1 / 2)<1\right)$ and that $\Gamma_{-}(x) \leq x$ for any $x \in \mathbb{R}_{+}$(as consequence of the second condition in (5.3)). Furthermore, we have that the limit in (5.1) holds.

(ii) Examples of functionals $F$ that satisfy condition (5.2) include a European call option payoff with strike $K \in \mathbb{R}_{+}$, where $F(\omega)=(\omega(T)-K)^{+}$, the time-average $F(\omega)=\frac{1}{T} \int_{0}^{T} \omega(s) \mathrm{d} s$, and the running maximum $F(\omega)=\sup _{s \in[0, T]} \omega(s)$.

(iii) We note that $\Upsilon_{\Delta}$ may be equivalently expressed in terms of $\Psi_{\Delta}$ and $\widehat{\Psi}_{\Delta}$ as

$$
\Upsilon_{\Delta}=\sup _{x \in\left(0, \frac{1}{2}\right]}\left|\frac{\Psi_{\Delta}(x)-x}{\Gamma_{+}(x / \Delta) \Delta}-1\right| \vee \sup _{x \in\left(0, \frac{1}{2}\right)}\left|\frac{x-\widehat{\Psi}_{\Delta}(x)}{\Gamma_{-}(x / \Delta) \Delta}-1\right|
$$

(iv) We next provide an example to show the necessity of scaling the probability distortions. For a given uniform partition $\pi=\pi_{\Delta}$ of $[0, T]$ with mesh $\Delta$, a probability distortion $\Psi$ and $a_{+}, a_{-} \in \mathbb{R}_{+} \backslash\{0\}$, let us consider the risk charge under the iterated spectral risk measure $\mathrm{S}$ corresponding to $\Psi$ of the following statistic $X^{(\pi)}$ of the jump-sizes of $L^{(\pi)}=\left(L^{(\pi), 1}, \ldots, L^{(\pi), k}\right)$ :

$$
X^{(\pi)}:=N_{\pi}^{+}-N_{\pi}^{-}, \quad N_{\pi}^{ \pm}=\#\left\{t \in \pi \backslash\{T\}: \sum_{i=1}^{k}\left(\Delta L_{t}^{(\pi), i}\right)^{ \pm}>a_{ \pm}\right\} .
$$

From the form (2.4), (2.5) of the solution of the BS $\Delta \mathrm{E}$ associated to the iterated spectral risk measure $S$, we have that $Z^{(\pi)}$ is given by

$$
\begin{aligned}
& Z_{t}^{(\pi)}(x)=z_{+}^{(\pi)}(x)-z_{-}^{(\pi)}(x), \quad z_{ \pm}^{(\pi)}(x)=I_{A_{ \pm}}(x), \\
& A_{ \pm}=\left\{z \in \mathbb{R}^{k} \backslash\{0\}: \sum_{i=1}^{k}\left(z_{i}\right)^{ \pm}>a_{ \pm}\right\} .
\end{aligned}
$$

As a consequence, we have from (3.9) in Proposition 3.9 that the driver function takes the form

$$
\begin{aligned}
\bar{g}_{\Delta}\left(t, Z_{t}^{(\pi)}\right) \Delta= & \mathrm{C}^{\Psi \circ\left(v^{(\pi)} \Delta\right), \widehat{\Psi} \circ\left(v^{(\pi)} \Delta\right)}\left(z_{+}^{(\pi)}(f)-z_{-}^{(\pi)}(f)\right) \\
& -\Delta \int_{\mathbb{R}^{k} \backslash\{0\}}\left(z_{+}^{(\pi)}(x)-z_{-}^{(\pi)}(x)\right) v^{(\pi)}(\mathrm{d} x) \\
= & \Psi\left(\mathbb{P}\left[\Delta L_{t_{1}}^{(\pi)} \in A_{+}\right]\right)-\mathbb{P}\left[\Delta L_{t_{1}}^{(\pi)} \in A_{+}\right] \\
& +\mathbb{P}\left[\Delta L_{t_{1}}^{(\pi)} \in A_{-}\right]-\widehat{\Psi}\left(\mathbb{P}\left[\Delta L_{t_{1}}^{(\pi)} \in A_{-}\right]\right) .
\end{aligned}
$$

For given $t \in \pi \backslash\{T\}$, the iterated spectral risk measure $\mathrm{S}_{t}\left(X^{(\pi)}\right)$ may therefore be expressed in terms of the functions $D_{\Delta}^{+}$and $D_{\Delta}^{-}:\left[0, \Delta^{-1}\right] \rightarrow \mathbb{R}_{+}$, given by $D_{\Delta}^{+}(x)=\Psi(x \Delta)-x$ and $D_{\Delta}^{-}(x)=x-\widehat{\Psi}(x \Delta)$, as 


$$
\begin{aligned}
\mathrm{S}_{t}\left(X^{(\pi)}\right)-\mathbb{E}\left[X^{(\pi)} \mid \mathcal{F}_{t}^{(\pi)}\right]= & \mathbb{E}\left[\sum_{s \geq t, s \in \pi \backslash\{T\}} \bar{g}_{\Delta}\left(s, Z_{s}^{(\pi)}\right) \Delta \mid \mathcal{F}_{t}^{(\pi)}\right] \\
= & (T-t)\left(\frac{1}{\Delta} D_{\Delta}^{+}\left(\Delta^{-1} \mathbb{P}\left[\Delta L_{t_{1}}^{(\pi)} \in A_{+}\right]\right)\right. \\
& \left.+\frac{1}{\Delta} D_{\Delta}^{-}\left(\Delta^{-1} \mathbb{P}\left[\Delta L_{t_{1}}^{(\pi)} \in A_{-}\right]\right)\right) .
\end{aligned}
$$

Note that as $\Delta \searrow 0, \Delta^{-1} \mathbb{P}\left[\Delta L_{t_{1}}^{(\pi)} \in A_{ \pm}\right] \rightarrow v\left(A_{ \pm}\right)$and

$$
\mathbb{E}\left[X^{(\pi)} \mid \mathcal{F}_{t}^{(\pi)}\right] \longrightarrow(T-t)\left(v\left(A_{+}\right)-v\left(A_{-}\right)\right)+N_{t}^{+}-N_{t}^{-},
$$

where $N_{t}^{ \pm}=\#\left\{s \in(0, t]: L_{s}-L_{s-} \in A_{ \pm}\right\}$. Hence, this suggests that for the sequence of iterated spectral risk measures to converge, $\Delta^{-1} D_{\Delta}^{+}(x)$ and $\Delta^{-1} D_{\Delta}^{-}(x)$ are to admit limits as $\Delta \searrow 0$.

Proof of Theorem 5.2 We note first that as $L^{(\pi)} \stackrel{\mathrm{d}}{\rightarrow} L$ when $\Delta \searrow 0, F\left(L^{(\pi)}\right)$ converges in distribution to $F(L)$, which is an element of $\mathcal{L}^{2}$. Furthermore, by Corollary 2.12, the collection $\left(F\left(L^{(\pi)}\right)^{2}\right)_{\pi}$ is uniformly integrable. Thus, in view of Theorem 2.14, it suffices to verify that the sequence of driver functions $\left(\bar{g}_{\Delta}\right)_{\Delta \in(0,1]}$ of the iterated spectral risk measures $\mathrm{S}^{\Delta}$ given in Proposition 3.9 satisfies Condition 2.13, which we proceed to do.

Let $t \in[0, T]$. Our first observation is that by subadditivity and nonnegativity of $\bar{g}_{\Delta}$, we have for any $h, \ell \in L^{2}\left(v^{(\pi)}\right)$ that

$$
\left|\bar{g}_{\Delta}(t, h)-\bar{g}_{\Delta}(t, \ell)\right| \leq \bar{g}_{\Delta}(t, h-\ell) \vee \bar{g}_{\Delta}(t, \ell-h),
$$

so that to verify Condition 2.13(i), it suffices to show that $\bar{g}_{\Delta}(t, h) /|h|_{2, \pi}$ is uniformly bounded. We have for any $\Delta \in(0,1]$ and $h \in L^{2}\left(v^{(\pi)}\right)$ that

$$
\begin{aligned}
\bar{g}_{\Delta}(t, h)= & \frac{1}{\Delta}\left(\mathrm{C}^{\Psi_{\Delta} \circ\left(v^{(\pi)} \Delta\right), \widehat{\Psi}_{\Delta} \circ\left(v^{(\pi)} \Delta\right)}(h)-\Delta \int h \mathrm{~d} v^{(\pi)}\right) \\
= & \frac{1}{\Delta}\left(\mathrm{C}_{+}^{\Psi_{\Delta} \circ\left(v^{(\pi)} \Delta\right)}\left(h^{+}\right)-\Delta \int h^{+} \mathrm{d} v^{(\pi)}\right) \\
& +\frac{1}{\Delta}\left(\Delta \int h^{-} \mathrm{d} v^{(\pi)}-\mathrm{C}_{+}^{\widehat{\Psi}_{\Delta} \circ\left(v^{(\pi)} \Delta\right)}\left(h^{-}\right)\right) \\
= & \mathrm{C}_{+}^{\Gamma_{+} \circ v^{(\pi)}}\left(h^{+}\right)+R^{\Delta}\left(h^{+}\right)+\mathrm{C}_{+}^{\Gamma_{-} \circ v^{(\pi)}}\left(h^{-}\right)+\widehat{R}^{\Delta}\left(h^{-}\right),
\end{aligned}
$$

where the remainder terms $R^{\Delta}\left(h^{+}\right)$and $\widehat{R}^{\Delta}\left(h^{-}\right)$are given in terms of the identity function $I:[0,1] \rightarrow[0,1], I(x)=x$, as

$$
\begin{aligned}
& R^{\Delta}\left(h^{+}\right)=\frac{1}{\Delta} \int_{0}^{\infty}\left(\left(\Psi_{\Delta}-I\right)\left(v^{(\pi)}\left(h^{+}>x\right) \Delta\right)-\Gamma_{+}\left(v^{(\pi)}\left(h^{+}>x\right)\right) \Delta\right) \mathrm{d} x, \\
& \widehat{R}^{\Delta}\left(h^{-}\right)=\frac{1}{\Delta} \int_{0}^{\infty}\left(\left(I-\widehat{\Psi}_{\Delta}\right)\left(v^{(\pi)}\left(h^{-}>x\right) \Delta\right)-\Gamma_{-}\left(v^{(\pi)}\left(h^{-}>x\right)\right) \Delta\right) \mathrm{d} x .
\end{aligned}
$$


Since $v^{(\pi)}\left(h^{ \pm}>x\right) \leq\left|h^{ \pm}\right|_{2, v^{(\pi)}}^{2} / x^{2}$ for $x \in \mathbb{R}_{+} \backslash\{0\}$ by Chebyshev's inequality, it follows that for

$$
x \geq H^{ \pm}:=\left|h^{ \pm}\right|_{2, v(\pi)} \sqrt{2 \Delta},
$$

the mass of $\Delta v^{(\pi)}\left(h^{ \pm}>x\right)$ is bounded above by $1 / 2$. Recalling the form of $\Upsilon_{\Delta}$ (see Remark 5.3 (iii)) and that $\Gamma_{+}+\Gamma_{-}$is bounded (by $\Gamma_{\infty}$, say), we have

$$
\begin{aligned}
\left|R^{\Delta}\left(h^{+}\right)\right| \leq & \Upsilon_{\Delta} \mathrm{C}_{+}^{\Gamma_{+} \circ v^{(\pi)}}\left(h^{+}\right) \\
& +\int_{0}^{H^{+}}\left(\Gamma_{+}\left(v^{(\pi)}\left(h^{+}>x\right)\right)+\Gamma_{-}\left(v^{(\pi)}\left(h^{-} \leq x\right)\right)\right) \mathrm{d} x \\
\leq & \Upsilon_{\Delta} \mathrm{C}_{+}^{\Gamma_{+} \circ v^{(\pi)}}\left(h^{+}\right)+H^{+} \Gamma_{\infty}, \\
\left|\widehat{R}^{\Delta}\left(h^{-}\right)\right| \leq & \Upsilon_{\Delta} \mathrm{C}_{+}^{\Gamma_{-} \circ v^{(\pi)}}\left(h^{-}\right)+H^{-} \Gamma_{\infty} .
\end{aligned}
$$

Combining (5.4)-(5.7) and the $K_{\Gamma_{+}}$- and $K_{\Gamma_{-}}$-Lipschitz-continuity of $\mathrm{C}_{+}^{\Gamma_{+} \circ v^{(\pi)}}$ and $\mathrm{C}_{-}^{\Gamma_{-} \circ v^{(\pi)}}$ (Proposition 3.5) and the fact that the values $\mathrm{C}_{+}^{\Gamma_{+} \circ v^{(\pi)}}(0)$ and $\mathrm{C}_{+}^{\Gamma_{-} \circ v^{(\pi)}}(0)$ are equal to 0 , we find

$$
\left|\bar{g}_{\Delta}(h)\right| \leq \tilde{C}|h|_{2, v(\pi)},
$$

where $\tilde{C}=\left(K_{\Gamma_{+}}+K_{\Gamma_{-}}+2 \sqrt{2} \Gamma_{\infty}\right)\left(1+\sup _{\Delta \in(0,1]} \Upsilon_{\Delta}\right)$ is finite by the limit (5.1) in Definition 5.1. This completes the proof of Condition 2.13(i).

We next turn to Condition 2.13(ii). Let $h$ be a continuous function with the property that $c_{h}:=\sup |h(x) / x| \in \mathbb{R}_{+}$. Since $v^{(\pi)}$ converges weakly to $v$, we have $v^{(\pi)}(h>x) \rightarrow v(h>x)$ at $x \in \mathbb{R}_{+} \backslash\{0\}$ that are points of continuity. Hence, as $\Gamma_{ \pm}$ are continuous, it follows that $\Gamma_{ \pm}\left(v^{(\pi)}(h>x)\right) \rightarrow \Gamma_{ \pm}(v(h>x))$ at such $x$. Next we show that the latter functions are dominated by an integrable function. By Chebyshev's inequality, $\Gamma_{ \pm}\left(v^{(\pi)}(h>x)\right) \leq \Gamma_{ \pm}\left(|h|_{2, v^{(\pi)}}^{2} / x^{2}\right)$, while it follows from the inequality (2.22) that $v_{2}^{(\pi)} \leq v_{2}$, where $v_{2}^{(\pi)}=\int_{\mathbb{R}^{k} \backslash\{0\}}|x|^{2} v^{(\pi)}(\mathrm{d} x)$. Hence we have the bound

$$
|h|_{2, v^{(\pi)}} \leq c_{h} \sqrt{v_{2}^{(\pi)}} \leq c_{h} \sqrt{v_{2}}
$$

Also, for any $d \in \mathbb{R}_{+}, \Gamma_{ \pm}\left(d^{2} / x^{2}\right)$ is integrable since

$$
\int_{d / \sqrt{\nu_{0}}}^{\infty} \Gamma_{ \pm}\left(d^{2} / x^{2}\right) \mathrm{d} x=K_{\Gamma_{ \pm}} d
$$

where $v_{0}=v\left(\mathbb{R}^{k} \backslash\{0\}\right)$ and $K_{\Gamma_{ \pm}}$is given in (3.1). As a consequence, the dominated convergence theorem gives that $\mathrm{C}_{+}^{\Gamma_{ \pm} \mathrm{ov}}{ }^{(\pi)}\left(h^{ \pm}\right) \rightarrow \mathrm{C}_{+}^{\Gamma_{ \pm} \mathrm{ov}}\left(h^{ \pm}\right)$as $\Delta \searrow 0$. Further, in view of (5.1), $R^{\Delta}\left(h^{+}\right)$and $\widehat{R}^{\Delta}\left(h^{-}\right)$tend to zero as $\Delta \searrow 0$. This establishes Condition 2.13(ii), and the proof is complete. 


\section{Dynamically optimal portfolio allocation}

We next consider dynamic portfolio problems concerning balancing gain and risk as quantified by the dynamic spectral risk measure (DSR). We suppose the investment horizon is $T>0$ and consider the DSR associated to the spectral driver function $\bar{g}$. In this section, we impose the following restriction on the Lévy measure $v$.

Assumption 6.1 The support of $v$ is included in $(-1, \infty)^{k}$.

We suppose that the financial market consists of a risk-free bond and $n$ risky stocks with discounted prices $\hat{S}=\left(\hat{S}^{1}, \ldots, \hat{S}^{n}\right)$ evolving according to the system of SDEs

$$
\begin{aligned}
\frac{\mathrm{d} \hat{S}_{t}^{i}}{\hat{S}_{t-}^{i}} & =b^{i} \mathrm{~d} t+\int_{\mathbb{R}^{k} \backslash\{0\}} R^{i} x \tilde{N}(\mathrm{~d} t \times \mathrm{d} x), \quad i=1, \ldots, n, t \in(0, T], \\
\hat{S}_{0} & =s_{0} \in\left(\mathbb{R}_{+} \backslash\{0\}\right)^{k},
\end{aligned}
$$

where $b^{i} \in \mathbb{R}$ is the excess log-return and $R^{i} \in \mathbb{R}^{k}$ is the (row) vector of jump coefficients with nonnegative coordinates that are such that $\left(R^{i}\right)^{\top} \mathbf{1} \leq 1$ (where $\mathbf{1} \in \mathbb{R}^{k}$ denotes the $k$-column vector of ones and $v^{\top}$ the transpose of a vector $v$ ). Given the form of the model, we have $\hat{S}_{t}^{i} \in \mathcal{L}_{t}^{2}$ and $\hat{S}_{t}^{i}>0$ for any $i=1, \ldots, k$ and $t \in[0, T]$.

Let us consider the case of a small investor whose trades have a negligible impact on prices and let us adopt the classical frictionless and self-financing setting (no transaction costs, infinitely divisible assets, continuous-time trading, no funds are infused into or withdrawn from the portfolio at intermediate times, etc.). At any time $t \in[0, T]$, the investor decides to allocate the fraction $\theta_{t}^{i}$ of the current wealth for investment into the stock $\hat{S}^{i}, i=1, \ldots, n$, so that if $X_{t-}^{\theta}$ denotes the discounted wealth just before time $t$, we have that $\theta_{t}^{i} X_{t-}^{\theta} / \hat{S}_{t-}^{i}$ is the number of stocks $i$ held in the portfolio at time $t$. We suppose that certain limits are placed on the leverage ratio of the portfolio and on the size of the short holdings in the various stocks, and that this restriction is phrased in terms of a bounded and closed set $\mathcal{B} \subset \mathbb{R}^{n}$ as the requirement that

$$
\theta_{t}(\omega) \in \mathcal{B} \quad \text { for any }(t, \omega) \in[0, T] \times \Omega \text {. }
$$

Example 6.2 To impose constraints on the fractions of the current wealth invested in the bond account and the stock accounts, we take

$$
\mathcal{B}=\left\{x \in \mathbb{R}_{+}^{n}: x_{i} \geq-L_{i}, \sum_{i=1}^{n} x_{i} \leq 1+L_{0}\right\}
$$

for some $L_{0}, \ldots, L_{n} \in \mathbb{R}_{+}$. In particular, by taking $L_{i}>0$, we impose a limit on the borrowing $(i=0)$ or the number of stock $i$ that may be shorted $(i \neq 0)$. The case of a "long-only" investor that has no short sales and only invests his own wealth (no borrowing) corresponds to taking $L_{0}=L_{1}=\cdots=L_{n}=0$. 
We call an allocation strategy $\theta=\left(\theta_{t}\right)_{t \in[0, T]}$ admissible if $\theta$ is predictable and (6.1) holds. We denote by $\mathcal{A}$ the collection of admissible allocation strategies. Denoting by $R=\left(R^{i}\right)_{i=1, \ldots, k}$ the $\mathbb{R}^{n \times k}$-matrix with $i$ th row equal to $R^{i}$, we have that the discounted value $X^{\theta}=\left(X_{t}^{\theta}\right)_{t \in[0, T]}$ of a portfolio corresponding to $\theta \in \mathcal{A}$ evolves according to the SDE

$$
\begin{aligned}
\frac{\mathrm{d} X_{t}^{\theta}}{X_{t-}^{\theta}} & =\theta_{t}^{\top} b \mathrm{~d} t+\int_{\mathbb{R}^{k} \backslash\{0\}} \theta_{t}^{\top} R x \tilde{N}(\mathrm{~d} t \times \mathrm{d} x), \quad t \in\left(0, \tau^{\theta} \wedge T\right], \\
X_{0}^{\theta} & =x \in \mathbb{R}_{+} \backslash\{0\}, \quad X_{t}^{\theta}=X_{\tau^{\theta} \wedge T}^{\theta}, \quad t \in\left(\tau^{\theta} \wedge T, T\right],
\end{aligned}
$$

where $\tau^{\theta}=\inf \left\{t \in[0, T]: X_{t}^{\theta}<0\right\}$ (with $\left.\inf \emptyset=+\infty\right)$ is the first time that the value of the portfolio becomes negative, when the investor has to stop trading.

\subsection{Portfolio optimisation under dynamic spectral risk measures}

We consider next the stochastic optimisation problem given in terms of DSR by the criterion to minimise, for $t \in[0, T]$, the quantity

$$
\tilde{\mathcal{J}}_{t}^{\theta}=\rho_{t}^{\bar{g}}\left(X_{T \wedge \tau^{\theta}}^{\theta}\right)
$$

The investor's problem is to identify a stochastic process $\tilde{\mathcal{J}}^{*}=\left(\tilde{\mathcal{J}}_{t}^{*}\right)_{t \in[0, T]}$ and an allocation strategy $\theta^{*} \in \mathcal{A}$ such that

$$
\tilde{\mathcal{J}}_{t}^{*}=\underset{\theta \in \mathcal{A}}{\operatorname{essinf}} \tilde{\mathcal{J}}_{t}^{\theta}=\tilde{\mathcal{J}}_{t}^{\theta^{*}}, \quad t \in[0, T] .
$$

While the problem in (6.2) may be solved via a BSDE approach (as used for instance in $[5,36]$ to analyse utility optimisation and robust portfolio choice problems), due to its Markovian nature, it may also be approached via classical methods based on an associated Hamilton-Jacobi-Bellman equation; this is the method we present here. One class of allocation strategies are those of feedback type, defined as follows.

Definition 6.3 Denote by $\tilde{\Theta}$ the set of functions $\bar{\theta}:[0, T] \times \mathbb{R}_{+} \rightarrow \mathcal{B}$ that are such that there exists a unique solution $X^{\bar{\theta}}=\left(X_{t}^{\bar{\theta}}\right)_{t \in[0, T]}$ to the SDE

$$
\begin{aligned}
\frac{\mathrm{d} X_{t}^{\bar{\theta}}}{X_{t-}^{\bar{\theta}}} & =\bar{\theta}\left(t, X_{t-}^{\bar{\theta}}\right)^{\top} b \mathrm{~d} t+\bar{\theta}\left(t, X_{t-}^{\bar{\theta}}\right)^{\top} R x \tilde{N}(\mathrm{~d} t \times \mathrm{d} x), \quad t \in\left(0, \tau^{\bar{\theta}}\right], \\
X_{0}^{\bar{\theta}} & =x, \quad X_{t}^{\bar{\theta}}=X_{\tau^{\bar{\theta}} \wedge T}^{\bar{\theta}}, \quad t \in\left(\tau^{\bar{\theta}} \wedge T, T\right],
\end{aligned}
$$

where $\tau^{\bar{\theta}}=\inf \left\{t \in[0, T]: X_{t}^{\bar{\theta}}<0\right\}$. A strategy $\theta \in \mathcal{A}$ is called a feedback allocation strategy if there exists a feedback function $\bar{\theta} \in \bar{\Theta}$ such that

$$
\theta_{t}=\bar{\theta}\left(\tau^{\bar{\theta}} \wedge t, X_{\tau^{\bar{\theta}} \wedge(t-)}^{\overline{\bar{\theta}}}\right), \quad t \in[0, T],
$$

where $X_{0-}^{\bar{\theta}}=X_{0}^{\bar{\theta}}$ and $X^{\bar{\theta}}$ solves the SDE in (6.3) and (6.4). 
Associated to a given allocation strategy $\bar{\theta} \in \bar{\Theta}$ of feedback type, there exists a value function $V^{\bar{\theta}}$ satisfying $J_{t}^{*}=V^{\bar{\theta}}\left(t, X_{t}^{\bar{\theta}}\right)$ for $t \in[0, T]$ (as a consequence of the Markov property). If sufficiently regular, the function $V^{\bar{\theta}}$ satisfies a semilinear PIDE that is given in terms of certain operators $\mathcal{D}^{\theta}$ and $\mathcal{G}^{\theta}$ indexed by $\theta \in \mathcal{B}$. For any function $f \in C^{1,1}([0, T] \times \mathbb{R})$, these operators yield the functions $\mathcal{D}_{t, x}^{\theta} f: \mathbb{R}^{k} \rightarrow \mathbb{R}$ and $\mathcal{G}^{\theta} f:[0, T] \times \mathbb{R}_{+} \backslash\{0\} \rightarrow \mathbb{R}$ given in terms of

$$
b_{\theta}=\theta^{\top} b, \quad R_{\theta}=\theta^{\top} R, \quad \theta \in \mathcal{B},
$$

by (denoting $f^{\prime}=\frac{\partial f}{\partial x}$ )

$$
\begin{aligned}
\left(\mathcal{D}_{t, x}^{\theta} f\right)(y) & =f\left(t, x+x R_{\theta} y\right)-f(t, x), \\
\mathcal{G}^{\theta} f(t, x) & =b_{\theta} f^{\prime}(t, x)+\int_{\mathbb{R}^{k} \backslash\{0\}}\left(\left(\mathcal{D}_{t, x}^{\theta} f\right)(y)-f^{\prime}(t, x) x R_{\theta} y\right) v(\mathrm{~d} y) .
\end{aligned}
$$

The nonlinear Feynman-Kac formula (see Remark 2.7) implies that if the following semilinear PIDE has a sufficiently regular solution, it is equal to $V^{\bar{\theta}}$ :

$$
\begin{aligned}
\dot{v}(t, x)+\mathcal{G}^{\bar{\theta}(t, x)} v(t, x)+\bar{g}\left(\mathcal{D}_{t, x}^{\bar{\theta}(t, x)} v\right) & =0, \quad(t, x) \in[0, T) \times \mathbb{R}_{+} \backslash\{0\}, \\
v(t, x) & =-x, \quad(t, x) \in[0, T) \times \mathbb{R}_{-}, \\
v(T, x) & =-x, \quad x \in \mathbb{R} .
\end{aligned}
$$

Standard arguments suggest that if the optimal allocation strategy $\theta^{*}$ is of feedback type and the corresponding value function $V$ is sufficiently regular, then $V$ satisfies the Hamilton-Jacobi-Bellman (HJB) equation

$$
\begin{aligned}
\dot{V}(t, x)+\inf _{\theta \in \mathcal{B}}\left(\mathcal{G}^{\theta} V(t, x)+\bar{g}\left(\mathcal{D}_{t, x}^{\theta} V\right)\right) & =0, \quad(t, x) \in[0, T) \times \mathbb{R}_{+} \backslash\{0\}, \\
V(t, x) & =-x, \quad t \in[0, T) \times \mathbb{R}_{-}, \\
V(T, x) & =-x, \quad x \in \mathbb{R} .
\end{aligned}
$$

Next we verify that a sufficiently smooth solution of the HJB equation gives rise to a solution of the optimisation problem in (6.2). Let $C_{b}^{1,1}([0, T] \times \mathbb{R})$ denote the set of $C^{1,1}$-functions $f:[0, T] \times \mathbb{R} \rightarrow \mathbb{R}$ with bounded first order derivatives.

Theorem 6.4 Let $w \in C_{b}^{1,1}([0, T] \times \mathbb{R})$ be a solution of the HJB equation (6.6)-(6.8) and let the function $\tilde{\theta}:[0, T] \times \mathbb{R}_{+} \rightarrow \mathcal{B},(t, x) \mapsto \tilde{\theta}(t, x)$, given by

$$
\tilde{\theta}(t, x) \in \underset{\theta \in \mathcal{B}}{\arg \sup }\left(\mathcal{G}^{\theta} w(t, x)+\bar{g}\left(\mathcal{D}_{t, x}^{\theta} w\right)\right)
$$

be such that $\tilde{\theta} \in \bar{\Theta}$. Then the feedback strategy $\tilde{\theta}^{*}=\left(\tilde{\theta}_{t}^{*}\right)_{t \in[0, T]}$ with feedback function $\tilde{\theta}$ is optimal for (6.2) and we have $\tilde{\mathcal{J}}_{t}^{*}=\tilde{\mathcal{J}}_{t}^{\tilde{\theta}^{*}}=w\left(t, X_{t \wedge \tau^{\tilde{\theta}}}\right)$, where $X^{\tilde{\theta}}$ solves the SDE in (6.3) and (6.4) with $\bar{\theta}$ replaced by $\tilde{\theta}$. 
Proof Letting $\theta \in \mathcal{A}$ be an arbitrary admissible strategy, $t<\tau^{\theta} \wedge T$ and $w$ as in the theorem, we find by an application of Itô's lemma that

$$
\begin{aligned}
& w\left(T \wedge \tau^{\theta}, X_{T \wedge \tau^{\theta}}^{\theta}\right)-w\left(t, X_{t}^{\theta}\right)+\int_{t}^{T \wedge \tau^{\theta}} \bar{g}\left(\mathcal{D}^{\theta_{s}} w_{s, X_{s}^{\theta}}\right) \mathrm{d} s \\
& =\int_{t}^{T \wedge \tau^{\theta}}\left(\left(\dot{w}+\mathcal{G}^{\theta_{s}} w\right)\left(s, X_{s}^{\theta}\right)+\bar{g}\left(\mathcal{D}^{\theta_{s}} w_{s, X_{s}^{\theta}}\right)\right) \mathrm{d} s+M_{T \wedge \tau^{\theta}}^{\theta}-M_{t}^{\theta},
\end{aligned}
$$

where $M^{\theta}$ is the square-integrable martingale given by

$$
\begin{aligned}
M_{t}^{\theta}= & \int_{0}^{t} w^{\prime}\left(s, X_{s-}^{\theta}\right)\left(\mathrm{d} X_{s}^{\theta}-b_{\theta_{s}} X_{s}^{\theta} \mathrm{d} s\right) \\
& +\int_{0}^{t} \int_{\mathbb{R}^{k} \backslash\{0\}}\left(\mathcal{D}^{\theta_{s}} w_{s, X_{s}^{\theta}}(y)-w^{\prime}\left(s, X_{s}^{\theta}\right) x R_{\theta_{s}} y\right) \tilde{N}(\mathrm{~d} s \times \mathrm{d} y) .
\end{aligned}
$$

Note that by the HJB equation (6.6), the first term on the right-hand side of (6.9) is nonpositive. Hence by taking conditional expectations in (6.9) and using (6.7) and (6.8), we have that

$$
w\left(t, X_{t}^{\theta}\right) \leq \mathbb{E}\left[-X_{T \wedge \tau^{\theta}}^{\theta}+\int_{t}^{T \wedge \tau^{\theta}} \bar{g}\left(\mathcal{D}^{\theta_{s}} w_{s, X_{s}^{\theta}}\right) \mathrm{d} s \mid \mathcal{F}_{t}\right]=\mathcal{J}_{t}^{\theta} .
$$

Since $\theta \in \mathcal{A}$ is arbitrary, we have that

$$
w\left(t, X_{t}^{\bar{\theta}}\right) \leq \underset{\theta \in \mathcal{A}}{\operatorname{essinf}} \mathcal{J}_{t}^{\theta}=\mathcal{J}_{t}^{*} .
$$

If we choose $\theta=\tilde{\theta}^{*}$, we note that the first term on the right-hand side of (6.9) vanishes and the inequalities in (6.10), (6.11) become equalities, so that $\mathcal{J}_{t}^{*}=w\left(t, X_{t}^{\tilde{\theta}^{*}}\right)$. As the process $X^{\tilde{\theta}^{*}}$ coincides with the process $X^{\tilde{\theta}}$ solving the SDE in (6.3) and (6.4), the proof is complete.

\subsubsection{Case of a "long-only" investor}

We next restrict to the case of a "long-only" investor (see Example 6.2). In this case, we note that for any admissible allocation strategy $\theta \in \mathcal{A}$, the solvency constraint $X_{t}^{\theta} \in \mathbb{R}_{+}$is satisfied for all $t \in[0, T]$ so that $\tau^{\theta}=\infty$ a.s. We identify the optimal strategy as follows.

Theorem 6.5 Let $\theta^{*} \in \mathcal{B}$ satisfy

$$
\theta^{*} \in \underset{\theta \in \mathcal{B}}{\arg \sup }\left(b_{\theta}-\bar{g}\left(-R_{\theta} I\right)\right),
$$

where $b_{\theta}$ and $R_{\theta}$ are given in (6.5) and $I: \mathbb{R}^{k} \rightarrow \mathbb{R}^{k}$ is given by $I(y)=y$. Then $\tilde{\theta}^{*}=\left(\tilde{\theta}_{t}^{*}\right)_{t \in[0, T]}$ given by $\tilde{\theta}_{t}^{*} \equiv \theta^{*}$ is an optimal strategy and

$$
\mathcal{J}_{t}^{*}=-X_{t}^{\theta^{*}} \exp \left((T-t)\left(b_{\theta^{*}}-\bar{g}\left(-R_{\theta^{*}} I\right)\right)\right) .
$$


Proof The assertions follow by an application of the verification theorem (Theorem 6.4). We note first that as the function $\theta \mapsto b_{\theta}-\bar{g}\left(-R_{\theta} I\right)$ is concave, it attains its maximum on the compact set $\mathcal{B}$. Thus, the set in (6.12) is not empty and $\theta^{*}$ is well defined. Moreover, given the positive homogeneity of $g$, it is straightforward to verify that the function $C:[0, T] \rightarrow \mathbb{R}$ given by

$$
C(t)=-\exp \left((T-t)\left(b_{\theta^{*}}-\bar{g}\left(-R_{\theta^{*}} I\right)\right)\right)
$$

satisfies the ODE

$$
\begin{aligned}
\dot{C}(t)+\inf _{\theta \in \mathcal{B}}\left(b_{\theta} C(t)+\bar{g}\left(C(t) R_{\theta} I\right)\right) & =0, \quad t \in[0, T), \\
C(T) & =-1 .
\end{aligned}
$$

As a consequence, we have that the candidate value function $V:[0, T] \times \mathbb{R}_{+} \rightarrow \mathbb{R}_{+}$ given by $V(t, x)=C(t) x$ satisfies the HJB equation (6.6)-(6.8) (here we used again the positive homogeneity of $\bar{g}$ ). The assertions follow now by an application of Theorem 6.4.

Acknowledgements MP's research supported in part by EPSRC Platform Grant EP/I019111/1. Research carried out in part at KdV Institute, University of Amsterdam, supported by NWO-STAR (MP), and at Tilburg University, supported by NWO-VENI (MS).

We thank two anonymous referees and an Associate Editor for careful reading and useful comments. We thank H. Albrecher, J. Blanchet, P. Jevtić, R. Laeven, H. Schumacher, J. Sekine and participants of the London-Paris Bachelier Workshop (Paris), Workshop on Mathematical Finance and Related Issues (Osaka), SF@W seminar (Warwick), Workshop on Advanced Modelling in Mathematical Finance (Kiel), and Workshop on Models and Numerics in Financial Mathematics (Leiden) for useful discussions and suggestions. A previous version of the paper was entitled "On consistent valuations based on distorted expectations: from multinomial walks to Lévy processes".

Open Access This article is distributed under the terms of the Creative Commons Attribution 4.0 International License (http://creativecommons.org/licenses/by/4.0/), which permits unrestricted use, distribution, and reproduction in any medium, provided you give appropriate credit to the original author(s) and the source, provide a link to the Creative Commons license, and indicate if changes were made.

\section{References}

1. Acerbi, C.: Spectral measures of risk: a coherent representation of subjective risk aversion. J. Bank. Finance 26, 1505-1518 (2002)

2. Artzner, P., Delbaen, F., Eber, J.M., Heath, D.: Coherent measures of risk. Math. Finance 9, 203-228 (1999)

3. Artzner, P., Delbaen, F., Eber, J.M., Heath, D., Ku, H.: Coherent multiperiod risk adjusted values and Bellman's principle. Ann. Oper. Res. 152, 5-22 (2007)

4. Barles, G., Buckdahn, R., Pardoux, E.: Backward stochastic differential equations and integral-partial differential equations. Stoch. Stoch. Rep. 60, 57-83 (1997)

5. Becherer, D.: Bounded solutions to backward SDEs with jumps for utility optimization and indifference hedging. Ann. Appl. Probab. 16, 2027-2054 (2006)

6. Bion-Nadal, J.: Dynamic risk measures: time consistency and risk measures from BMO martingales. Finance Stoch. 12, 219-244 (2008)

7. Bion-Nadal, J.: Time consistent dynamic risk measures. Stoch. Process. Appl. 119, 633-654 (2009)

8. Carlier, G., Dana, R.A.: Core of convex distortions of a probability. J. Econ. Theory 113, 199-222 (2003) 
9. Chen, Z., Epstein, L.: Ambiguity, risk, and asset returns in continuous time. Econometrica 70, 1403$1443(2002)$

10. Cheridito, P., Delbaen, F., Kupper, M.: Dynamic monetary risk measures for bounded discrete-time processes. Electron. J. Probab. 11, 57-106 (2006)

11. Cherny, A.S.: Weighted V@R and its properties. Finance Stoch. 10, 367-393 (2006)

12. Cherny, A.S.: Capital allocation and risk contribution with discrete-time coherent risk. Math. Finance 19, 13-40 (2009)

13. Cohen, S., Elliott, R.: A general theory of finite state backward stochastic difference equations. Stoch. Process. Appl. 120, 442-466 (2010)

14. Cohen, S., Elliott, R.: Backward stochastic difference equations and nearly time-consistent nonlinear expectations. SIAM J. Control Optim. 49, 125-139 (2011)

15. Coquet, F., Hu, Y., Mémin, J., Peng, S.: Filtration-consistent nonlinear expectations and related gexpectations. Probab. Theory Relat. Fields 123, 1-27 (2002)

16. Delbaen, F.: The structure of $\mathrm{m}$-stable sets and in particular of the set of risk neutral measures. In: Emery, M., Yor, M. (eds.) In Memoriam Paul-André Meyer. Séminaire de Probabilités XXXIX. Lecture Notes in Mathematics, vol. 1874, pp. 215-258. Springer, Berlin (2006)

17. Delong, Ł.: Backward Stochastic Differential Equations with Jumps and Their Actuarial and Financial Applications. Springer, Berlin (2013)

18. Denneberg, D.: Non-Additive Measure and Integral. Kluwer Academic, Dordrecht (1994)

19. Dolinsky, Y., Nutz, M., Soner, H.M.: Weak approximation of $G$-expectation. Stoch. Process. Appl. 122, 664-675 (2012)

20. Duffie, D., Epstein, L.G.: Stochastic differential utility. Econometrica 60, 353-394 (1992)

21. Eberlein, E., Madan, D.B., Pistorius, M., Yor, M.: Bid and ask prices as non-linear continuous time G-expectations based on distortions. Math. Financ. Econ. 8, 265-289 (2014)

22. Elliott, R.J., Siu, T.K., Cohen, S.N.: Backward stochastic difference equations for dynamic convex risk measures on a binomial tree. J. Appl. Probab. 52, 771-785 (2015)

23. Epstein, L.G., Schneider, M.: Recursive multiple-priors. J. Econ. Theory 113, 1-31 (2003)

24. Epstein, L.G., Zin, S.E.: Substitution, risk aversion, and the temporal behavior of consumption and asset returns: a theoretical framework. Econometrica 57, 937-969 (1989)

25. Föllmer, H., Penner, I.: Convex risk measures and the dynamics of their penalty functions. Stat. Decis. 24, 61-96 (2006)

26. Föllmer, H., Schied, A.: Stochastic Finance, 3rd edn. de Gruyter, Berlin (2011)

27. Hansen, L.P., Sargent, T.J.: Robustness. Princeton University Press, Princeton (2008)

28. Hardy, M.R., Wirch, J.L.: The iterated CTE: a dynamic risk measure. N. Am. Actuar. J. 8, 62-75 (2004)

29. Jacod, J., Shiryaev, A.N.: Limit Theorems for Stochastic Processes. Springer, Berlin (1987)

30. Jobert, A., Rogers, L.: Valuations and dynamic convex risk measures. Math. Finance 18, 1-22 (2008)

31. Klöppel, S., Schweizer, M.: Dynamic indifference valuation via convex risk measures. Math. Finance 17, 599-627 (2007)

32. Koopmans, T.C.: Stationary ordinal utility and impatience. Econometrica 28, 287-309 (1960)

33. Kreps, M.K., Porteus, E.L.: Temporal resolution of uncertainty and dynamic choice theory. Econometrica 46, 185-200 (1978)

34. Kusuoka, S.: On law-invariant coherent risk measures. Adv. Math. Econ. 3, 83-95 (2001)

35. Kusuoka, S., Morimoto, Y.: Homogeneous law-invariant coherent multi-period value measures and their limits. J. Math. Sci. Univ. Tokyo 14, 117-156 (2007)

36. Laeven, R.J.A., Stadje, M.: Robust portfolio choice and indifference valuation. Math. Oper. Res. 39, 1109-1141 (2014)

37. Madan, D., Pistorius, M., Stadje, M.: Convergence of BS $\Delta$ Es driven by random walks to BSDEs: the case of (in)finite activity jumps with general driver. Stoch. Process. Appl. 126, 1553-1584 (2016)

38. Peng, S.: A generalized dynamic programming principle and Hamilton-Jacobi-Bellman equation. Stoch. Stoch. Rep. 38, 119-134 (1992)

39. Peng, S.: Nonlinear expectations, nonlinear evaluations, and risk measures. In: Frittelli, M., Runggaldier, W. (eds.) Stochastic Methods in Finance. Lecture Notes in Mathematics, pp. 165-254. Springer, Berlin (2004)

40. Riedel, F.: Dynamic coherent risk measures. Stoch. Process. Appl. 112, 185-200 (2004)

41. Roorda, B., Schumacher, J.M.: Time consistency conditions for acceptability measures-with an application to Tail Value at Risk. Insur. Math. Econ. 40, 209-230 (2007)

42. Rosazza Gianin, E.: Risk measures via g-expectations. Insur. Math. Econ. 39, 19-34 (2006) 
43. Royer, M.: Backward stochastic differential equations with jumps and related non-linear expectations. Stoch. Process. Appl. 116, 1358-1376 (2006)

44. Sato, K.: Lévy Processes and Infinitely Divisible Distributions. Cambridge University Press, Cambridge (1999)

45. Stadje, M.: Extending dynamic convex risk measures from discrete time to continuous time: a convergence approach. Insur. Math. Econ. 47, 391-404 (2010)

46. Strotz, R.H.: Myopia and inconsistency in dynamic utility optimisation. Rev. Econ. Stud. 23, 165-180 (1955)

47. Tutsch, S.: Update rules for convex risk measures. Quant. Finance 8, 833-843 (2008)

48. Wang, S.: Premium calculation by transforming the layer premium density. ASTIN Bull. 26, 71-92 (1996)

49. Weber, S.: Distribution-invariant risk measures, information, and dynamic consistency. Math. Finance 16, 419-442 (2006) 